\title{
Amplitude stabilization of micromechanical oscillators using engineered nonlinearity
}

\author{
James M. L. Miller, ${ }^{1,2, *}$ Ariosto Gomez-Franco, ${ }^{2}$ Dongsuk D. Shin, ${ }^{2}$ Hyun-Keun Kwon, ${ }^{2}$ and Thomas W. Kenny ${ }^{2, \dagger}$ \\ ${ }^{1}$ Department of Mechanical Science and Engineering, University of Illinois at Urbana-Champaign, Urbana, Illinois 61801, USA \\ ${ }^{2}$ Departments of Mechanical and Electrical Engineering, Stanford University, Stanford, California 94305, USA
}

(Received 22 December 2020; accepted 9 August 2021; published 20 September 2021)

\begin{abstract}
Micromechanical oscillators provide periodic output signals for clocks and sensors by vibrating in a single mechanical mode. The mode is conventionally excited into self-sustained oscillations and stabilized with an external electronic feedback loop. A paradigm is emerging for sustaining vibrations by coupling the mechanical mode with internal degrees of freedom, such as photons, electrons, or auxiliary mechanical modes. An open question in these hybrid vibrational systems is the corresponding internal sources of nonlinearity that can stabilize the oscillations, and their impact on oscillator performance. Here, we delineate two kinds of amplitude-stabilization mechanisms in micromechanical oscillators, geometric nonlinear damping and repulsive contact, and show that these mechanisms can coexist in the same device and their interplay and resonance frequency stability can be tuned in situ by adjusting the feedback strength. An auxiliary source of viscous dissipation and nonlinear dissipation accompanies the repulsive contact, which stabilizes the amplitude during sidewall collisions. The onset of self-sustained oscillations yields distinct spectral-temporal signatures that can be used to identify the amplitude stabilization nonlinearities.
\end{abstract}

DOI: 10.1103/PhysRevResearch.3.033268

\section{INTRODUCTION}

Micro- and nanoelectromechanical (MEM/NEM) resonators often serve as the frequency-determining elements within oscillators for resonant sensors and timing references. The oscillations are conventionally sustained by monitoring the vibrations near the resonance frequency and applying an external phase-shifted feedback force [1]. A host of effective damping techniques are emerging, which utilize the interaction between the mechanical mode and photons [2-5], electrons [6,7], magnons [8,9], or auxiliary mechanical modes [10-12]. By eliminating the external feedback loop, these coupling mechanisms could enable MEM/NEM oscillators with potentially better performance and suitability to different operating conditions than conventional oscillators. The interaction dynamics and effective damping tuning achievable with these emerging coupling mechanisms have received a great deal of attention in recent years [13]. Utilizing these techniques for novel oscillator topologies opens up a parallel challenge that has received relatively less attention: understanding and controlling the nonlinear dynamics of the mechanical oscillations. There is ongoing work to harness nonlinearities to improve oscillator performance, including utilizing zero-dispersion points [14-16] and modal coupling for frequency stabilization $[17,18]$, and reservoir engineering

\footnotetext{
*jmlm@illinois.edu

†tkenny@stanford.edu
}

Published by the American Physical Society under the terms of the Creative Commons Attribution 4.0 International license. Further distribution of this work must maintain attribution to the author(s) and the published article's title, journal citation, and DOI.
$[19,20]$ for storing energy in auxiliary mechanical modes. For self-sustained oscillators based on hybrid vibrational systems, the nonlinearities are integral to performance [21]. Additionally, ongoing efforts to operate MEM/NEM resonators in their quantum regime [22-27], with ultranarrow linewidths [28-30], opens up a path to harness nonlinearities to develop quantum bits (qubits) based on mechanical resonators [31-33].

When pumping any coupling mechanism beyond the selfoscillation threshold, the effective damping of the mechanical mode becomes negative and the amplitude grows rapidly until stiffening nonlinearity [21] or nonlinear damping [34,35] stabilizes the vibrations. Stiffening nonlinearity is readily available in resonator geometries that experience midplane stretching of the vibrating element relative to the undeformed frame [36], such as doubly clamped beams [37,38] and membranes $[39,40]$. Many other important geometries, such as cantilevers [41,42] and bulk acoustic wave resonators $[43,44]$, exhibit exceedingly weak stiffening nonlinearity. Capacitive transduction schemes compound this problem in MEM/NEM resonators since electrostatics contribute thirdand higher-order softening nonlinearities $[45,46]$, which will destabilize the oscillations [47]. Positive nonlinear damping provides another route for stabilizing the oscillation amplitude, and geometric nonlinear dissipation has been observed in MEM/NEM resonators constructed from doubly clamped beams [35,48-50] and membranes [51,52]. The origins of intrinsic positive nonlinear dissipation in MEM/NEM resonators are under investigation [53-56], and there is evidence of negative nonlinear dissipation arising in directly [57-59] and parametrically [60] driven micromechanical resonators. A third possible amplitude stabilization mechanism, repulsive contact, utilizes the interactions between the vibrating mechanical element and an adjacent electrode. Squeezing of 

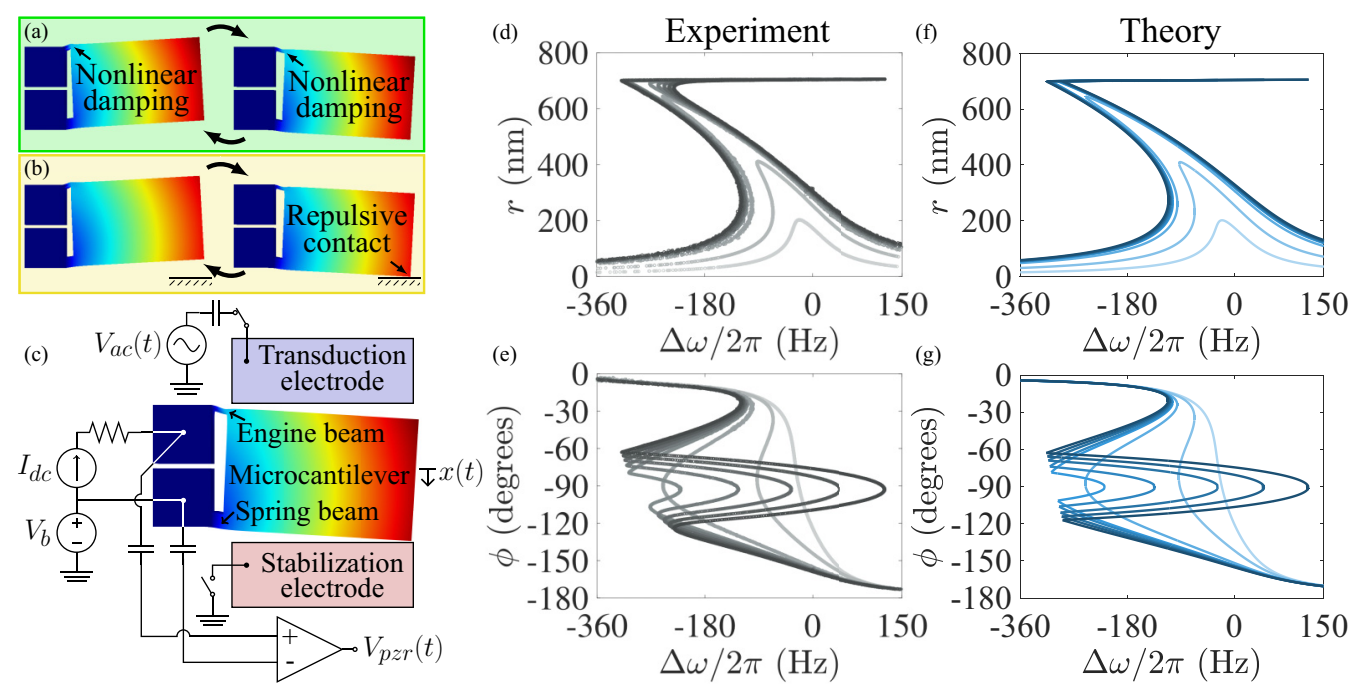

FIG. 1. Characterization of the nonlinear electromechanical resonators. (a) A depiction of amplitude stabilization in a micromechanical cantilever via nonlinear dissipation in a high-stress connecting element. (b) An analogous depiction to (a) for stabilization via repulsive contact with an adjacent electrode. (c) A simplified schematic of the micromechanical resonator, showing the vibration modeshape, electrical biasing, and displacement detection scheme. The device is voltage biased at $V_{b}$, and a current over-bias is applied between the two anchors to flow a direct current $\left(I_{\mathrm{dc}}\right)$ through the resonator. The motion is read out piezoresistively $\left(V_{\mathrm{pzr}}\right)$, or capacitively. An external harmonic drive $\left(V_{\mathrm{ac}}\right)$ signal can be alternately applied to an adjacent electrode to characterize the forced response. [(d),(e)] The amplitude and phase of the forced response vs frequency detuning $\Delta \omega=\omega-\omega_{0}$ for a repulsive-contact-stabilized resonator, Device $\mathrm{D}(L=50 \mu \mathrm{m}, g=700 \mathrm{~nm})$. The harmonic drive voltage varies from $V_{\mathrm{ac}}=100 \mathrm{mV}$ to $V_{\mathrm{ac}}=370 \mathrm{mV}$. [(f),(g)] The corresponding theoretical amplitude and phase curves of the device measured in [(d), (e)], using the nonlinear model for the amplitude and phase in Eqs. (4) and (5), respectively.

the gas film in the gap at large amplitudes can contribute additional dissipation, and the Hertzian-like contact of the asperities on the contacting surfaces can contribute stiffening nonlinearity. Repulsive contact is ubiquitous in the context of atomic force microscopy [61,62], whereby the competing effects of van der Waals attractive forces and Hertzian repulsive forces on the microcantilever tip influence the vibration dynamics.

Here we investigate the geometric nonlinear dissipation and repulsive contact mechanisms as a resource for amplitude stabilization in MEM/NEM oscillators. Geometric nonlinear dissipation, as depicted in Fig. 1(a), may arise in regions of a resonator that experience significant stress. Repulsive contact, as depicted in Fig. 1(b), stems from the nonlinear interactions between the resonator and an adjacent surface. Using a common device architecture, we isolate independent sources of dissipation corresponding to each of these two amplitude stabilization mechanisms and confirm that these differing sources of dissipative nonlinearity are responsible for the amplitude stabilization in both cases. Unlike standard microcantilevers vibrating in a diving-board mode, the cantilevered geometry in this study is designed to concentrate axial stress in a narrow anchoring beam to near the single-crystal-silicon fracture limit during oscillations. By adjusting the length of the supporting beams and the width of the capacitive gaps on either side of the cantilever, the relative contributions of the intrinsic nonlinear dissipation in silicon and the external nonlinearity of the repulsive contact with the sidewalls can be controlled. We use geometric nonlinear dissipation to stabilize the oscillation amplitude for over 100 million cycles, observe slow-time amplitude fluctuations for feedback strengths near threshold, and identify silicon fatigue as the primary con- sideration for long-term oscillator operation. For amplitude stabilization via repulsive contact, we demonstrate stable operation for over 1 billion cycles, and show that significant improvements in oscillator frequency stability can be attained by utilizing a feedback strength close to threshold. We show that repulsive contact is a robust mechanism for stable operation of oscillators within hybrid vibrational systems based on electron coupling, and can be readily implemented for other domains of coupling and for a wide variety of other flexural and bulk mode resonator geometries.

\section{DEVICES}

We investigate the geometric nonlinear damping and repulsive contact amplitude stabilization mechanisms using silicon piezoresistive microcantilevers, as depicted in Fig. 1(c). The oscillations are sustained by leveraging the internal coupling between the mechanics, electrons, and heating known as thermal-piezoresistive pumping $[63,64]$. This recently discovered electrothermal feedback scheme is under investigation for novel mass sensors [65], signal pre-amplifiers for resonant sensors [66], and nanoscale narrow-band filters [67]. This internal feedback scheme enables us to tune the feedback strength beyond threshold by simply adjusting the direct current through the resonator, and eliminates the need to apply any external harmonic signals during self-sustained oscillations.

The devices are fabricated in a wafer-scale encapsulation process $[68,69]$, which produces stable microscale resonators in a hermetic vacuum-sealed environment. The ultraclean environment, stable material properties, and amenability to resonator construction makes this fabrication process ideal 
for studying nonlinear nanomechanics in silicon. The base of the cantilever is split into a wide "spring" beam and a narrow "engine" beam, which is a modification of the early thermal flexure actuator geometry [70,71]. The spring beam contributes most of the mechanical stiffness for the flexural modes of the cantilever, while the engine beam induces the self-sustained oscillations and contributes geometric nonlinear dissipation at large stresses [47]. The cantilevers have a thickness of $60 \mu \mathrm{m}$, and support a proof mass of $400 \mu \mathrm{m}$ length by $100 \mu \mathrm{m}$ width. The resonators are fabricated from n-type, moderately antimony doped single-crystal-silicon $\left(N_{d} \approx 4 \times 10^{18} \mathrm{~cm}^{-3}\right)$, and aligned in the $\langle 100\rangle$-direction for maximum longitudinal piezoresistive coefficients. The devices are voltage-biased with respect to an adjacent electrode to enable capacitive motion detection, and current-biased for the dual purposes of inducing self-sustained oscillations and reading out the motion piezoresistively. We utilize a custom transimpedance amplifier (TIA) to measure the capacitive signal, and a custom differential four-probe piezoresistive buffer to measure the piezoresistive signal. The measurement amplifiers are optimized to remain in their linear regime during the large-amplitude oscillations, while contributing as little noise as possible. The electrode on the engine beam side of the device, labeled the "transduction electrode" in Fig. 1(c), is connected to either the capacitive TIA for self-oscillation measurements or a signal generator for forced vibrations. The electrode on the spring beam side of the device, labeled the "stabilization electrode" in Fig. 1(c), is left floating during this study.

\section{THEORY}

The displacement $x$ of a driven single vibrational degree of freedom with linear stiffness, viscous damping, and an arbitrary nonlinear force can be described by

$$
\ddot{x}+\gamma \dot{x}+\omega_{0}^{2} x=f \cos (\omega t)+f_{\mathrm{NL}}(x, \dot{x}),
$$

where $\omega_{0}=\sqrt{k / m}$ is the angular resonance frequency, $k$ is the lumped stiffness, $m$ is the lumped mass, $\gamma=\omega_{0} / Q$ is the resonance linewidth, $Q$ is the quality factor, $f$ is the mass-normalized drive force, and $\omega$ is the angular drive frequency. We include a contribution to the nonlinear force $f_{\mathrm{NL}}$ from Duffing (Kerr) nonlinearity, a quintic amplitudefrequency nonlinearity, nonlinear dissipation, and repulsive contact forces as

$$
f_{\mathrm{NL}}(x, \dot{x})=-\alpha x^{3}-\beta x^{5}-\eta x^{2} \dot{x}+f_{c}(x, \dot{x}),
$$

where $\alpha$ is the Duffing parameter, $\beta$ is the quintic stiffness parameter, and $\eta$ is the nonlinear damping parameter. $\alpha$ and $\beta$ are the coefficients of third-order and fifth-order amplitudefrequency nonlinearities, respectively, because they are the proportionality factors for forces that scale as $x^{3}$ and $x^{5}$. The $\alpha$ and $\beta$ terms vary the resonator stiffness and the $\eta$ term varies the resonator damping with amplitude. These nonlinear terms are phenomenological, and are often used to model the dynamics of nonlinear oscillators $[21,47,60]$. In the devices measured here, $\omega_{0} / 2 \pi$ varies from $300 \mathrm{kHz}$ to $500 \mathrm{kHz}, \gamma$ varies from $100 \mathrm{rad} \mathrm{s}^{-1}$ to $300 \mathrm{rad} \mathrm{s}^{-1}$, $\alpha \approx-10^{22} \operatorname{rad}^{2} \mathrm{~m}^{-2} \mathrm{~s}^{-2}, \beta \approx-10^{34} \mathrm{rad}^{2} \mathrm{~m}^{-4} \mathrm{~s}^{-2}$, and $\eta \approx 10^{15} \mathrm{rad}^{2} \mathrm{~m}^{-2} \mathrm{~s}^{-1}$ [47]. $\alpha$ and $\beta$ are negative because of electrostatic softening from the adjacent electrodes. The tipsidewall force can assume a variety of constitutive relations $[61,72,73]$. Here we consider a piecewise viscous damping term, a piecewise nonlinear damping term, and a piecewise linear stiffness term arising from the repulsive contact:

$$
f_{c}(x, \dot{x})= \begin{cases}0, & x<g, \\ -\gamma_{c} \dot{x}-\eta_{c}[x-g]^{2} \dot{x}-\omega_{c}^{2}[x-g], & x \geqslant g,\end{cases}
$$

where $g$ is the gap size, $\gamma_{c}$ is the contact viscous damping parameter, $\eta_{c}$ is the contact nonlinear damping parameter, and $\omega_{c}^{2}=k_{c} / m$ is the mass-normalized contact stiffness. The piecewise stiffness in the contact force $f_{c}$ can be obtained from a first-order expansion of the Hertzian contact force in Derjaguin, Muller, Toporov, and Maugis (DMT-M) theory (see the Supplemental Material [74]). The attractive electrostatic and van der Waals force contribution is included up to fifth-order in $\alpha$ and $\beta$ within Eq. (2). We model the steadystate vibrations as $x=r \cos (\tau)$, where $\tau=\omega t+\phi$ is the slow time; $\tau$ tracks the envelope of the vibrations, and is slow compared to the real time variable $t$.

\section{A. Forced vibrations}

The amplitude $r$ and phase $\phi$ curves of the forced $\left(f>0 \mathrm{~N} \mathrm{~kg}^{-1}\right)$ resonator are given by

$$
\begin{gathered}
r(\omega, r)=\frac{f}{\sqrt{\left[a_{1}(\omega, r)\right]^{2}+\left[b_{1}(\omega, r)\right]^{2}}}, \\
\phi(\omega, r)=-\operatorname{atan}\left(\frac{b_{1}(\omega, r)}{a_{1}(\omega, r)}\right),
\end{gathered}
$$

where

$$
\begin{gathered}
a_{1}(\omega, r)=\omega_{0}^{2}\left[1-I_{\text {odd }}(\omega, r)\right]-\omega^{2}, \\
b_{1}(\omega, r)=\gamma \omega+\omega_{0}^{2} I_{\text {even }}(\omega, r) .
\end{gathered}
$$

The nonlinear force is accounted for in the period-averaged Fourier series components [62] $I_{\text {even }}$ and $I_{\text {odd }}$. These terms depend upon the vibration amplitude, and thus Eq. (4) in this formulation is defined implicitly in terms of the vibration amplitude and must be solved numerically. In the limiting case of linear oscillations, $I_{\mathrm{even}}$ and $I_{\text {odd }}$ go to zero and Eqs. (4) and (5) describe a driven simple harmonic oscillator. The Fourier series terms for the nonlinear force in Eq. (2) are given by

$$
\begin{gathered}
I_{\text {even }}(\omega, r)=\frac{\eta r^{2} \omega}{4 \omega_{0}^{2}}+I_{\text {even }, c 1}(\omega, r)+I_{\text {even }, c 2}(\omega, r), \\
I_{\text {odd }}(\omega, r)=-\frac{3 \alpha r^{2}}{4 \omega_{0}^{2}}-\frac{5 \beta r^{4}}{8 \omega_{0}^{2}}+I_{\text {odd }, c}(\omega, r), \\
I_{\text {even }, c 1}(\omega, r \geqslant g)=\frac{\gamma_{c} \omega}{2 \pi \omega_{0}^{2}}\left[2 \tau_{1}-\sin \left(2 \tau_{1}\right)\right], \\
I_{\text {even }, c 2}(\omega, r \geqslant g)=\mathfrak{C}_{1}+\mathfrak{C}_{2}+\mathfrak{C}_{3}+\mathfrak{C}_{4}+\mathfrak{C}_{5}, \\
I_{\text {odd }, c}(\omega, r \geqslant g)=-\frac{\omega_{c}^{2}}{\pi \omega_{0}^{2}}\left[\tau_{1}-\frac{2 g}{r} \sin \left(\tau_{1}\right)+\frac{\sin \left(2 \tau_{1}\right)}{2}\right],
\end{gathered}
$$


where

$$
\begin{aligned}
& \mathfrak{C}_{1}=\frac{\omega \eta_{c} \tau_{1}}{\pi \omega_{0}^{2}}\left[\frac{r^{2}}{4}+g^{2}\right], \\
& \mathfrak{C}_{2}=-\frac{\omega \eta_{c} g r \sin \left(\tau_{1}\right)}{\pi \omega_{0}^{2}}, \\
& \mathfrak{C}_{3}=-\frac{\omega \eta_{c} g^{2} \sin \left(2 \tau_{1}\right)}{2 \pi \omega_{0}^{2}}, \\
& \mathfrak{C}_{4}=\frac{\omega \eta_{c} g r \sin \left(3 \tau_{1}\right)}{3 \pi \omega_{0}^{2}}, \\
& \mathfrak{C}_{5}=-\frac{\omega \eta_{c} r^{2} \sin \left(4 \tau_{1}\right)}{16 \pi \omega_{0}^{2}} .
\end{aligned}
$$

$I_{\text {even }, c}$ and $I_{\mathrm{odd}, c}$ are zero when the vibration amplitude is smaller than the gap size $r<g$. For vibrations larger than the gap size, $\tau_{1}=\operatorname{acos}(g / r)$ characterizes the fraction of each vibration cycle that the resonator interacts with the sidewall. $I_{\text {even }}$ and $I_{\text {odd }}$ are dimensionless and describe the dissipative and conservative nonlinear forces, respectively.

\section{B. Self-sustained vibrations}

Beyond the self-oscillation threshold, the effective damping in the vibrational mode becomes negative [13], and the amplitude grows exponentially until nonlinearities stabilize the steady-state vibrations. The method of averaging can be utilized to predict the vibration envelope in the transient regime and the steady-state regime [47]. Considering Eq. (1), setting the external drive force $f$ and the contact force $f_{c}$ to zero, and applying the method of averaging, yields two averaged equations for the amplitude $r$ and phase $\phi$ :

$$
\begin{gathered}
\dot{r}=-\frac{\gamma_{\mathrm{eff}} r}{2}-\frac{\eta r^{3}}{8}, \\
\dot{\phi}=\frac{\left(\omega_{0}^{2}-\omega^{2}\right)}{2 \omega}+\frac{3 \alpha r^{2}}{8 \omega}+\frac{5 \beta r^{4}}{16 \omega} .
\end{gathered}
$$

where we denote the effective damping parameter by $\gamma_{\text {eff }}$. The first term on the right-hand side of Eq. (18) governs the exponential growth $\left(\gamma_{\mathrm{eff}}<0 \mathrm{rad} \mathrm{s}^{-1}\right)$ or decay $\left(\gamma_{\mathrm{eff}}>0 \mathrm{rad}\right.$ $\mathrm{s}^{-1}$ ) of the vibration amplitude. We note that the effective damping equals the actual resonator damping, $\gamma_{\text {eff }}=\gamma$, only in the absence of feedback or pumping. At the onset of self-sustained oscillations $\left(\gamma_{\mathrm{eff}}<0 \mathrm{rad} \mathrm{s}^{-1}\right)$, the vibrations are driven by thermal fluctuations, and thus the second term on the right-hand side of Eq. (18) can be neglected for the values of nonlinear damping that are characteristic of MEM/NEM resonators. The resulting linear first-order differential equation yields an exponential growth of vibration amplitude with time $t$ :

$$
r(t)=r_{0} \exp \left(-\frac{\gamma_{\mathrm{eff}} t}{2}\right) \approx \sqrt{\frac{k_{B} T}{m \omega_{0}^{2}}} \exp \left(-\frac{\gamma_{\mathrm{eff}} t}{2}\right),
$$

where $\gamma_{\text {eff }}$ is assumed to be constant and effectively negative starting at $t=0 \mathrm{~s}, k_{B}=1.3806 \times 10^{-23} \mathrm{~J} \mathrm{~K}^{-1}$ is Boltzmann's constant, and $T$ is the thermal bath temperature.

After the initial exponential ring-up, the vibration amplitude is stabilized by the nonlinear damping and/or the wall collisions. The steady-state oscillations corresponds to $\dot{r}=$
$0 \mathrm{~m} \mathrm{~s}^{-1}$ and yields

$$
r_{s s}=\left\{\begin{array}{ll}
2 \sqrt{-\gamma_{\mathrm{eff}} / \eta}, & r<g \\
g, & r \geqslant g
\end{array} .\right.
$$

In order to stabilize the self-oscillation amplitude without wall collisions, the geometric nonlinear damping must be positive and sufficiently large for the feedback strength used.

\section{FORCED VIBRATIONS}

We use forced vibrations to identify the relevant nonlinearities in our microcantilevers during repulsive contact. We disconnect the capacitive readout from the transduction electrode and use this electrode to apply a harmonic force near resonance. We read out the motion piezoresistively, with a direct current $I_{\mathrm{dc}}=1 \mathrm{~mA}$, well below the self-oscillation threshold. We measure a device with a support beam length $L=50 \mu \mathrm{m}$ and a gap size $g=700 \mathrm{~nm}$; since the support beam length is much larger than the gap size, geometric nonlinear dissipation is negligible and repulsive contact controls the dynamics. Figures 1(d) and 1(e) depicts the measured forced response on a repulsive-contact-stabilized device, for increasing drive amplitude. For amplitudes below the gap size, the electrostatic softening and attractive van der Waals forces contribute softening nonlinearity. The sidewall collisions are accompanied by a dramatic stiffening nonlinearity, corresponding to the Hertzian repulsive contact forces exerted by the asperities on the contacting surfaces.

The solutions to Eqs. (4) and (5) for the repulsive-contactstabilized device are shown in Figs. 1(f) and 1(g). Negative $\alpha$ and $\beta$ values are sufficient to describe the nonlinear force for vibration amplitudes below the gap size; the nonlinear dissipation in the engine beam is negligible at the low stress in the $L=50 \mu \mathrm{m}$ device, and we thus set $\eta \approx 0 \mathrm{rad}^{2} \mathrm{~m}^{-2} \mathrm{~s}^{-1}$. For vibration amplitudes beyond the gap size, the linear piecewise stiffness is sufficient to capture the slope of the vibration amplitude vs drive amplitude. The model reveals that the conservative force alone is not sufficient to describe the measurements; viscous and nonlinear dissipative forces play an important role in restricting the frequency upshift during repulsive contact. We utilize a viscous contact force, with $\gamma_{c}>0 \mathrm{rad} \mathrm{s}^{-1}$, and a nonlinear dissipative contact force, with $\eta_{c}>0 \operatorname{rad}^{2} \mathrm{~m}^{-2} \mathrm{~s}^{-1}$, to yield agreement between the model and experiments in this regime.

\section{SELF-SUSTAINED VIBRATIONS}

We next study the impact of the geometric nonlinear damping and repulsive contact on the self-oscillation dynamics. Figure 2 presents measurements on three microcantilevers with varying support beam length and gap size, corresponding to the negligible repulsive contact, negligible geometric nonlinear damping, and an intermediate-nonlinearity case where both amplitude stabilization mechanisms are important. We disconnect the signal generator from the transduction electrode, and connect the capacitive readout to that electrode to monitor the motion capacitively. We flow a direct current beyond threshold to induce self-oscillations, while recording the voltage output from the piezorestive and capacitive readouts. 
Nonlinear damping

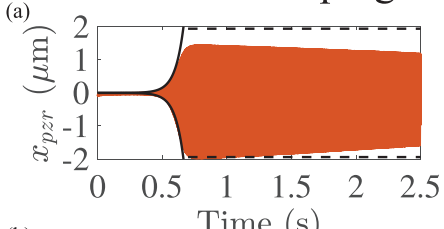

(b)

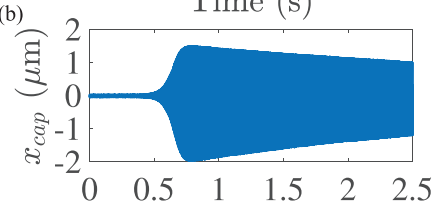

(c)
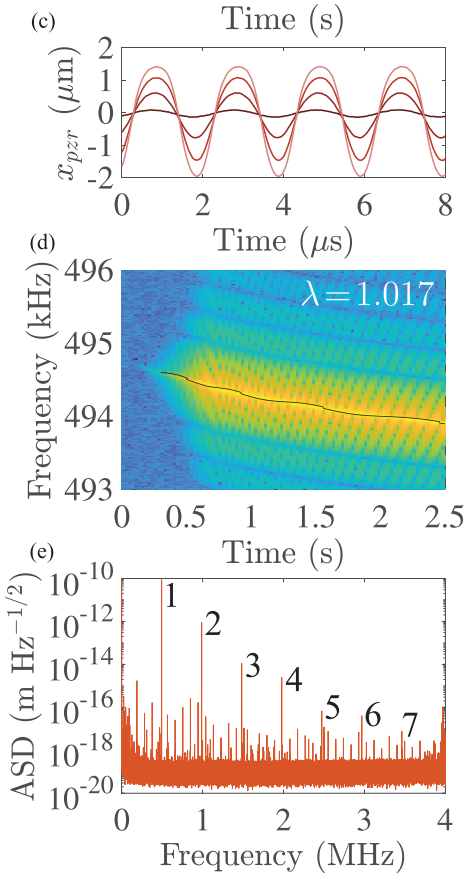

Intermediate nonlinearity
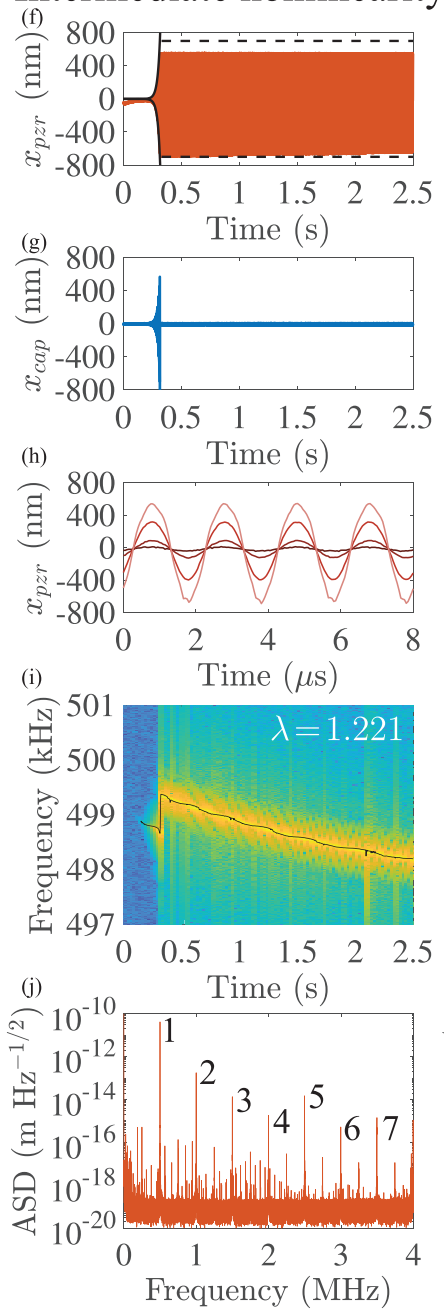
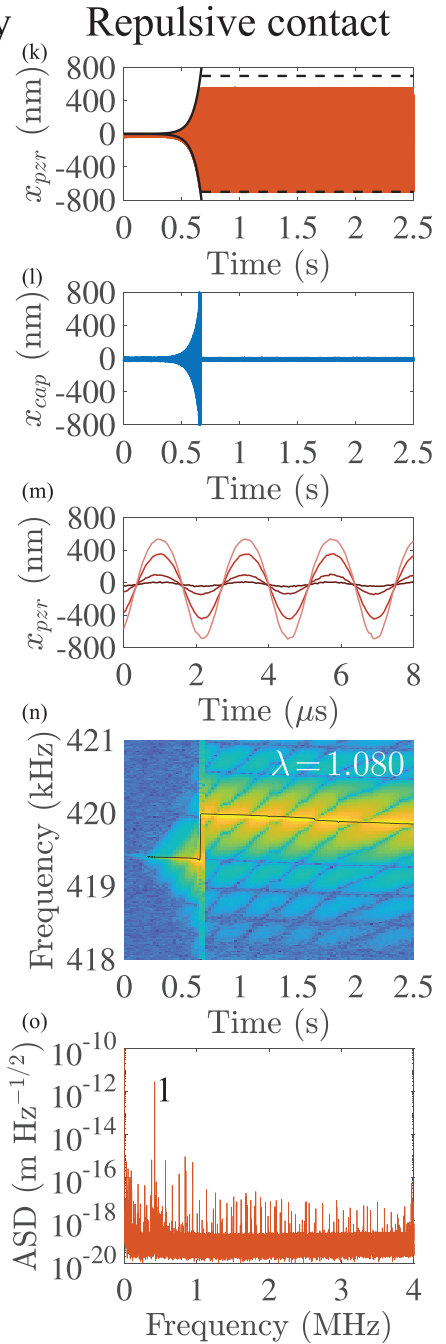

FIG. 2. The self-oscillation dynamics for three regimes of amplitude stabilization nonlinearities. (a) The measured self-oscillation waveform for the nonlinear-damping-stabilized oscillator, Device A $(L=6 \mu \mathrm{m}, g=2 \mu \mathrm{m})$, vs time after switching on the direct current, as measured using the piezoresistive readout $\left(x_{\mathrm{pzr}}\right)$. The exponential ring-up envelope from Eq. (20) (solid-black curves) and the steady-state amplitude from Eq. (21) (dashed-black curves) are overlaid. (b) The same measurement as (a), except measured using the capacitive readout $\left(x_{\text {cap }}\right)$. (c) The oscillation waveform at various points along the self-oscillation envelope, as measured using the piezoresistive readout. (d) A surface plot showing the Fast Fourier transforms near the resonance frequency during self-oscillations, with a 50-ms bin time. The black line plots the measured centroid frequency with a 5 -ms bin time. The feedback strength is characterized by $\lambda=I_{\mathrm{dc}} / I_{\mathrm{thresh}}$, where $I_{\text {thresh }}$ is the threshold current for self-oscillations. (e) The amplitude spectral density (ASD) of the steady-state oscillations, extracted from the piezoresistive output for a 1000-ms bin time. The fundamental peak and readout harmonics are labeled. [(f)-(j)] The same measurement as in [(a)-(e)], except for the intermediate-nonlinearity-stabilized oscillator, Device B $(L=6 \mu \mathrm{m}, g=700 \mathrm{~nm})$. [(k)-(o)] The same measurement as in [(a)-(e)], except for the repulsive-contact-stabilized oscillator, Device $\mathrm{C}(L=20 \mu \mathrm{m}, g=700 \mathrm{~nm})$. The feedback strength is $\lambda=1.017$ for the nonlinear-damping-stabilized oscillator in $[(\mathrm{a})-(\mathrm{e})], \lambda=1.221$ for the intermediate-nonlinearity-stabilized oscillator in [(f)-(j)], and $\lambda=1.080$ for the repulsive-contact-stabilized oscillator in $[(\mathrm{k})-(\mathrm{o})]$.

The self-sustained oscillations of the nonlinear-dampingstabilized microcantilever are studied in the first column of Fig. 2. This device has a support beam length $L=6 \mu \mathrm{m}$ and a gap size $g=2 \mu \mathrm{m}$, and thus the engine beam experiences stress approaching the fracture limit before the device contacts the sidewalls (see the Supplemental Material [74]) [75,76]. The short engine beam contributes geometric nonlinear damping at the large amplitudes of self-sustained oscillations [47]. The self-oscillation amplitude increases smoothly during the ring-up, as measured using the piezoresistive readout in Fig. 2(a) and using the capacitive readout in Fig. 2(b). The theoretical exponential growth envelope from Eq. (20) is overlaid in Fig. 2(a) and yields a good agreement with the measured envelope until the nonlinear damping begins to stabilize the vibrations, resulting in a smooth transition to the steady-state amplitude. The output waveform at various points along the self-oscillation envelope is shown in Fig. 2(c), and illustrates the anharmonicity in the piezoresistive response at large amplitudes. Fig. 2(d) presents the spectral dynamics of the self-oscillations near resonance via the Fast Fourier transforms (FFTs) of successive time bins of the piezoresistive response, which shows that the resonance frequency gradually decreases with time during self-sustained oscillations. The centroid frequency trace reveals a series of small down-shifts 
in the resonance frequency during the self-oscillations, potentially arising from fatigue in the engine beam. Figure 2(e) presents the spectrum of the steady-state oscillations recorded with the piezoresistive readout. A set of harmonics accompany the fundamental vibration peak, corresponding to the anharmonic waveform in Fig. 2(c), which could be induced by the engine beam nonlinearity that accompanies the strain levels that geometric nonlinear damping is appreciable at.

The self-sustained oscillations of the repulsive-contactstabilized microcantilever are studied in the third column of Fig. 2. This device has a support beam length $L=20 \mu \mathrm{m}$ and a gap size $g=700 \mathrm{~nm}$. The self-oscillation envelope, as measured using the piezoresistive readout in Fig. 2(k), abruptly plateaus once the device contacts the side electrodes. The transient amplitude matches the exponential growth from Eq. (20) up to the onset of wall collisions, at which point the amplitude is roughly constant and matches Eq. (21). Unlike the transient response of the nonlinear-damping-stabilized oscillator, there is no smooth transition away from exponential growth resulting from the second term on the right-hand side of Eq. (18). The corresponding capacitive readout measurement, presented in Fig. 2(1), registers a large motional current spike at the onset of repulsive contact, and ceases to accurately measure the vibrations; this corresponds to periodic electrical shorting to the transduction electrode. The piezoresistive waveform does not exhibit pronounced anharmonicity during oscillations stabilized by repulsive contact, as shown in Fig. 2(m), and there is not the set of harmonics in the selfoscillation spectrum in Fig. 2(o). These observations provide additional evidence that nonlinearity in the engine beam is not responsible for amplitude stabilization in this device. The measured FFTs in Fig. 2(n) provide clear evidence for the Hertzian stiffening and corresponding resonance frequency upshift during the onset of repulsive contact.

The self-sustained oscillations of the intermediatenonlinearity-stabilized microcantilever are studied in the middle column of Fig. 2. This device has a support beam length $L=6 \mu \mathrm{m}$ and a gap size $g=700 \mathrm{~nm}$, and the measurements reveal that both geometric nonlinear damping in the engine beam and repulsive contact with the sidewalls influences the dynamics. The measured FFTs in Fig. 2(i) display signatures of the repulsive contact, such as the large frequency upshift at the onset of steady-state oscillations, and also signatures of nonlinearity in the engine beam, such as the gradual reduction in the resonance frequency combined with a series of small downward kinks during self-oscillations. There are additionally harmonics in the piezoresistive readout self-oscillation spectrum, like for the nonlinear-dampingstabilized oscillator.

We next investigate the dynamics and stability in the nonlinear-damping-stabilized oscillator for varying feedback strength. The first column of Fig. 3 presents measurements at a large feedback strength, which causes the first few seconds of steady-state vibrations to be stabilized by wall collisions. After the initial period of sidewall collisions, the amplitude decreases and is stabilized by geometric nonlinear damping in the engine beam. The period of wall collisions is accompanied by substantial jitter in the amplitude envelope and resonance frequency, as Figs. 3(a) and 3(b) demonstrate. The amplitude stability and resonance frequency stability is much better in the nonlinear-damping-stabilized regime than in the wall collision regime, as the Allan deviation (ADEV) measurements in Figs. 3(c) and 3(d) show. The second column of Fig. 3 shows the measurements at a moderate feedback strength. The steady-state vibrations are completely stabilized by nonlinear damping in this regime, and the envelope amplitude is much smaller than the gap size. The amplitude envelope in Fig. 3(e) has a slow modulation with a period longer than 1 second. The amplitude stability and frequency stability in Figs. 3(g) and 3(h), respectively, both get worse relative to the nonlinear-damping-stabilized oscillations at a large feedback strength in Figs. 3(c) and 3(d). The third column of Fig. 3 shows the measurements at a feedback strength slightly above the self-oscillation threshold. The steady-state amplitude decreases, consistent with $\gamma_{\text {eff }}$ approaching zero in Eq. (21). The period of the slow modulation in the amplitude envelope in Fig. 3(i) also decreases to roughly $100 \mathrm{~ms}$, and is accompanied by an observable modulation in the standard deviation of the resonance frequency in Fig. 3(j), and a drop in amplitude and resonance frequency stability at a $100 \mathrm{~ms}$ averaging time in Figs. 3(k) and 3(l). The amplitude and resonance frequency stability both get worse relative to the nonlinear-dampingstabilized behavior at moderate and large feedback strength. In the drift-dominated regime of the Allan deviation $(\tau \approx 1 \mathrm{~s})$, the frequency stability improves with decreasing feedback strength.

We consider the dynamics and stability in the repulsivecontact-stabilized oscillator for varying feedback strength in Fig. 4. Reducing the feedback strength in Fig. 4(a) increases the ring-up time of the transient interval, like for the nonlinear-damping-stabilized device, but unlike the nonlinear-damping-stabilized device, the stabilized vibration amplitude is restricted by the gap size for all feedback strengths beyond threshold. The agreement between the vibration amplitudes for different direct currents after scaling by the current indicates that the piezoresistive output is linear for the repulsive-contact-stabilized devices. Because the geometric nonlinear damping is negligible for an engine beam length of $20 \mu \mathrm{m}$ and a gap-restricted vibration amplitude of $700 \mathrm{~nm}$, $r_{s s} \approx g \ll 2 \sqrt{-\gamma_{\text {eff }} / \eta}$, so the impact of feedback strength on the vibration amplitude envelope is not observable, unlike the nonlinear-damping-stabilized device. The standard deviation in resonance frequency of the steady-state vibrations at a $500 \mu$ s averaging time in Fig. 4(b) is roughly independent of feedback strength, as the corresponding resonance frequency stability measurements in Fig. 4(d) also indicate. For averaging times ranging from $1 \mathrm{~ms}$ to $10 \mathrm{~ms}$, reducing the feedback strength worsens the amplitude stability and resonance frequency stability. For averaging times beyond $100 \mathrm{~ms}$, where long-term drift starts to impact the stability, the stability measures in Figs. 4(c) and 4(d) improve with decreasing feedback strength. Fig. 4(e) shows the measured resonance frequency of the steady-state vibrations over a longer time period than in Fig. 4(b). The corresponding resonance frequency stability in Fig. 4(f) improves with decreasing feedback strength, consistent with Fig. 4(c) in the drift-dominated regime $(\tau=1 \mathrm{~s})$. The resonance frequency stability at $\tau=1 \mathrm{~s}$ for a given feedback strength is better in Fig. 4(f) than in Fig. 4(d) because the oscillations are given two minutes to stabilize before computing the resonance frequency Allan deviation in Fig. 4(f). 
(a)
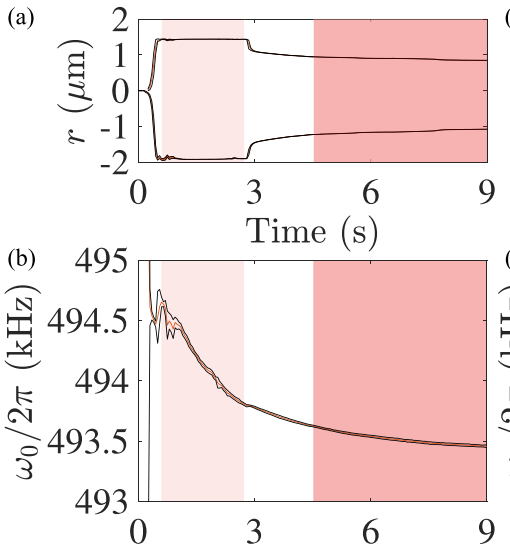

(c)

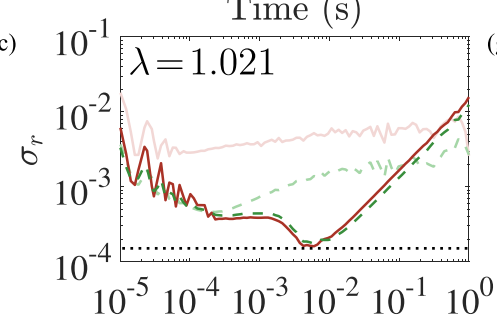

(d)

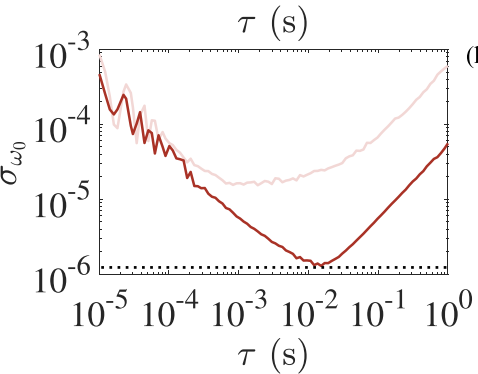

(e)
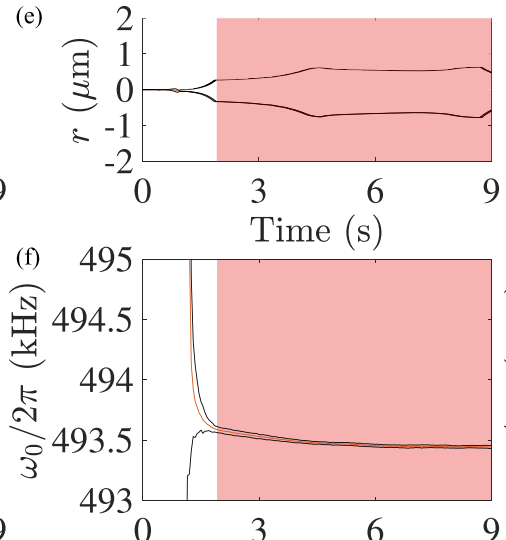

(g)

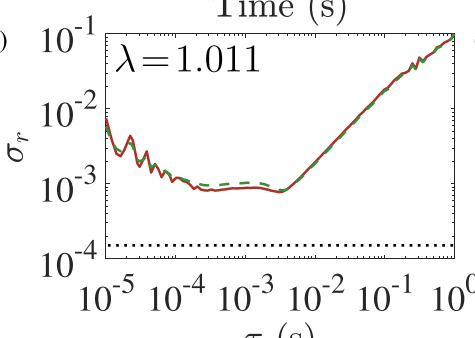

(i)
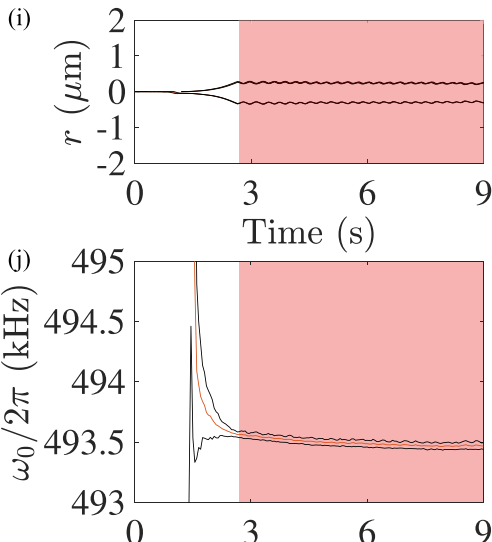

(k)

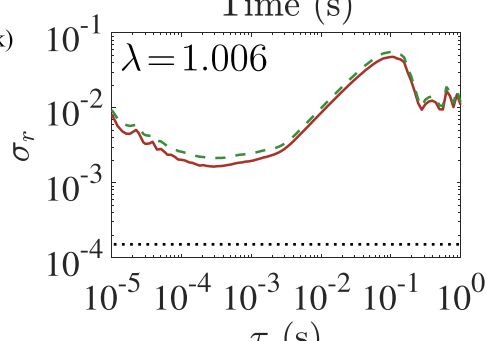

(h)

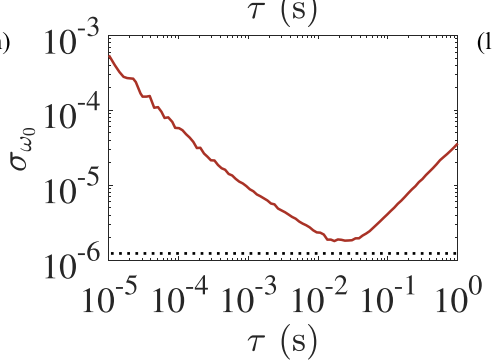

(1)

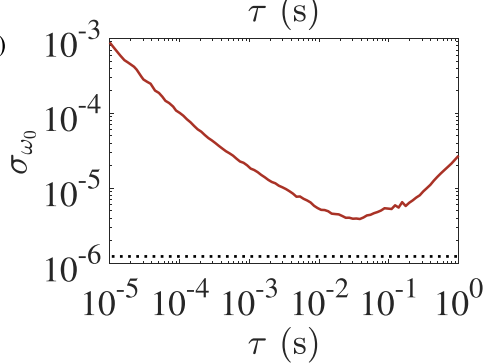

FIG. 3. The self-oscillation dynamics and stability for the nonlinear-damping-stabilized oscillator, Device A ( $L=6 \mu \mathrm{m}, g=2 \mu \mathrm{m}$ ), for decreasing feedback strength. (a) The measured mean (red curve) and \pm 2 standard deviations (black curves) of the amplitude $(r)$, using a 50-ms bin time and $\tau=500 \mu$ s averaging time. (b) The corresponding measured mean (red curve) and \pm 2 standard deviations (black curves) of the resonance frequency $\left(\omega_{0} / 2 \pi\right)$, as measured from the interpolated zero crossings, using a 50-ms bin time and $\tau=500 \mu$ s averaging time. (c) The extracted amplitude Allan deviation $\left(\sigma_{r}\right)$, for the lower (red curves) and upper (dashed-green curves) amplitude envelope. The light curves show the amplitude Allan deviation during the initial wall collision period, corresponding to the light red box in (a). The dark curves show the amplitude Allan deviation during the subsequent nonlinear-damping-stabilized period, corresponding to the dark red box in (a). The feedback strength for [(a)-(d)] is $\lambda=I_{\mathrm{dc}} / I_{\text {thresh }}=1.021$, where $I_{\text {thresh }}$ is the threshold current for self-oscillations. (d) The extracted resonance frequency Allan deviation $\left(\sigma_{\omega_{0}}\right)$. The light curves show the frequency Allan deviation during the initial wall collision period, corresponding to the light red box in (b). The dark curves show the frequency Allan deviation during the subsequent nonlinear-damping-stabilized period, corresponding to the dark red box in (b). $\tau$ corresponds to the integration time. [(e)-(h)] The same measurements as [(a)-(d)], except at a reduced feedback strength $\lambda=1.011$. The Allan deviations are only extracted from one time window, corresponding to the dark red boxes in $[(\mathrm{e})-(\mathrm{f})]$. [(i)-(l)] The same measurements as [(e)-(h)], except at a further reduced feedback strength $\lambda=1.006$.

\section{DISCUSSION}

Table I compares the stability measures for the nonlinear-damping-stabilized oscillator to the repulsivecontact-stabilized oscillator via the amplitude ADEV $\sigma_{r}$ and the resonance frequency ADEV $\sigma_{\omega_{0}}$ for feedback strengths well above threshold $(\lambda \gg 1)$ and slightly above threshold $(\lambda \approx 1)$. For large feedback strength, both measured minimum ADEVs between the two devices are comparable: $\sigma_{r, \min } \approx 10^{-4}$, and $\sigma_{\omega_{0}, \min } \approx 10^{-6}$. For feedback strength near threshold, the minimum ADEV measures improve for the repulsive-contact-stabilized oscillator but become significantly worse for the nonlinear-damping-stabilized oscillator. In the drift-dominated regime $(\tau=1 \mathrm{~s})$, both stability measures improve with decreasing feedback strength for both devices; reducing the feedback strength reduces the drift in amplitude and resonance frequency. This could be because at lower feedback strengths, the nonlinear-dampingstabilized oscillator experiences lower peak stress in the engine beam, and the repulsive-contact-stabilized oscillator impacts the sidewalls less forcefully; both effects would reduce the long-term drift in oscillator properties.

The minimum Allan deviation measures move in opposite directions with decreasing feedback strength for the nonlinear-damping-stabilized oscillator and the repulsive-contact-oscillator. Noise in the piezoresistive readout has an increasing effect on the stability measures for the nonlinear-damping-stabilized oscillator with decreasing 

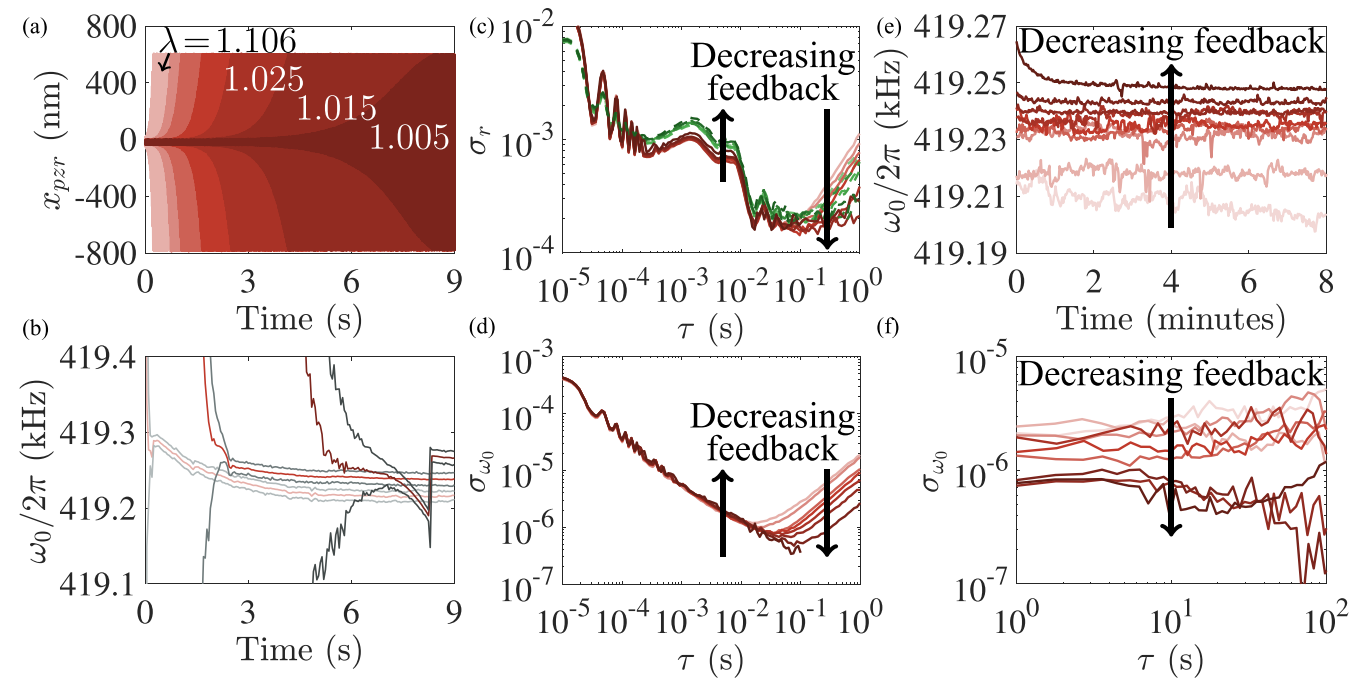

FIG. 4. The self-oscillation dynamics and stability for the repulsive-contact-stabilized oscillator, Device C $(L=20 \mu \mathrm{m}, g=700 \mathrm{~nm})$, for decreasing feedback strength. (a) The measured self-oscillation waveform vs time after switching on the direct current, as measured using the piezoresistive readout $\left(x_{\text {pzr }}\right)$, overlaid for decreasing feedback strength (light to dark red envelopes) beyond threshold. The feedback strength is characterized by $\lambda=I_{\mathrm{dc}} / I_{\text {thresh }}$, where $I_{\text {thresh }}$ is the threshold current for self-oscillations. The corresponding measured \pm 2 amplitude standard deviation curves lie on top of the mean amplitude for this plotting range. (b) The corresponding measured mean (red curve) and \pm 2 standard deviations (gray curves) of the resonance frequency $\left(\omega_{0} / 2 \pi\right)$, as measured from the interpolated zero crossings, using a $50 \mathrm{~ms}$ bin time and $\tau=500 \mu$ s averaging time, for feedback strengths $\lambda=1.106, \lambda=1.025$, and $\lambda=1.005$ (light- to dark-shade curves). (c) The extracted amplitude Allan deviation $\left(\sigma_{r}\right)$, for the lower (red curves) and upper (dashed-green curves) amplitude envelope, for decreasing feedback strength (light to dark shade curves), extracted from the steady-state vibrations in (a). (d) The extracted resonance frequency Allan deviation $\left(\sigma_{\omega_{0}}\right)$, for decreasing feedback strength (light- to dark-red curves), extracted from the steady-state vibrations in (a). (e) The steady-state selfoscillation frequency $\left(\omega_{0} / 2 \pi\right)$ for a longer time period after the initial transient interval, for decreasing feedback strength (light- to dark-red curves), as measured using a frequency counter. (f) The resonance frequency Allan deviation $\left(\sigma_{\omega_{0}}\right)$ corresponding to the time window from 2 to 8 minutes in (e) for decreasing feedback strength (light to dark red curves).

feedback strength, because the steady-state vibration amplitude decreases. Additionally, close to threshold the nonlineardamping-stabilized oscillator becomes more sensitive to fluctuations in resonator properties, such as viscous damping, thermal-piezoresistive feedback strength, and nonlinear

TABLE I. A comparison of stability measures between the nonlinear-damping-stabilized oscillator (Device A) and the repulsive-contact-stabilized oscillator (Device C).

\begin{tabular}{lcc}
\hline \hline Parameter & Device A & Device C \\
\hline$\omega_{0} /(2 \pi)[\mathrm{kHz}]$ & 494 & 419 \\
$Q\left(\times 10^{3}\right)$ & 23 & 25 \\
Beam length, $L[\mu \mathrm{m}]$ & 6 & 20 \\
Gap size, $g[\mathrm{~nm}]$ & 2000 & 700 \\
$\sigma_{r, \min , \lambda \gg 1}\left(\times 10^{-4}\right)$ & 1.6 & 1.7 \\
$\tau_{r, \min , \lambda \gg 1}[\mathrm{~ms}]$ & 6.0 & 17 \\
$\sigma_{\omega_{0}, \min , \lambda \gg 1}\left(\times 10^{-7}\right)$ & 13 & 12 \\
$\tau_{\omega_{0}, \min , \lambda \gg 1}[\mathrm{~ms}]$ & 15 & 15 \\
$\sigma_{r, \min , \lambda \approx 1}\left(\times 10^{-4}\right)$ & 16 & 1.6 \\
$\tau_{r, \min , \lambda \approx 1}[\mathrm{~ms}]$ & 0.29 & 33 \\
$\sigma_{\omega_{0}, \min , \lambda \approx 1}\left(\times 10^{-7}\right)$ & 39 & 3.4 \\
$\tau_{\omega_{0}, \min , \lambda \approx 1}[\mathrm{~ms}]$ & 39 & 69 \\
$\sigma_{r, \lambda \gg 1}(1 \mathrm{~s})\left(\times 10^{-4}\right)$ & 160 & 11 \\
$\sigma_{r, \lambda \approx 1}(1 \mathrm{~s})\left(\times 10^{-4}\right)$ & 110 & 2.9 \\
$\sigma_{\omega_{0}, \lambda \gg 1}(1 \mathrm{~s})\left(\times 10^{-7}\right)$ & 560 & 190 \\
$\sigma_{\omega_{0}, \lambda \approx 1}(1 \mathrm{~s})\left(\times 10^{-7}\right)$ & 270 & 27 \\
\hline \hline
\end{tabular}

damping. These fluctuations are visible in the modulation of the steady-state vibration envelope for the nonlineardamping-stabilized oscillator near threshold, as Fig. 3(i) and the corresponding amplitude ADEV in Fig. 3(k) at a 100-ms averaging time indicate. For the repulsive-contact-stabilized oscillators, the feedback strength does not influence the mean steady-state oscillation amplitude since the amplitude is restricted by the the wall collisions, as Fig. 4(a) demonstrates. The repulsive-contact-stabilized oscillator is thus less sensitive to fluctuations in device properties such as feedback strength near threshold. The properties of the wall collisions instead have a large impact on the oscillator performance. Decreasing the feedback strength results in less forceful wall collisions, which corresponds to lower oscillator drift, as the measured resonance frequency in Fig. 4(e) and the ADEV measurements in Figs. 4(d) and 4(f) suggest. The decreased drift with reducing feedback strength offsets the worse stability for averaging times ranging from $1 \mathrm{~ms}$ to $10 \mathrm{~ms}$, resulting in overall lower minimum ADEV values for the repulsive-contact-stabilized oscillator near threshold than far above threshold.

The nonlinear-damping-stabilized device measurements provide several indicators of the large operating stresses in the engine beam, including intrinsic nonlinear damping, fatigue, and anharmonic piezoresistive output waveform. The nonlinear damping was previously measured in this geometry using a parametric-resonance-based technique [47], and clearly limits the vibration amplitude since the nonlinear restoring force 
is softening [21] and there is no electrical shorting to the capacitive readout during oscillations in Fig. 2(b). The fatigue in the engine beam manifests itself in the self-sustained oscillator through the gradually decreasing oscillation frequency and vibration amplitude with time. The anharmonicity in the piezoresistive readout waveform is clearly more pronounced for the nonlinear-damping-stabilized device in Fig. 2(c) than for the intermediate-nonlinearity device in Fig. 2(h) or the repulsive-contact device in Fig. 2(m). Considering Eq. (21), variation in the internal feedback strength is probably the source for the gradual decrease in steady-state amplitude in Fig. 3(a) and the long-term fluctuating amplitude in Figs. 3(e) and 3(i). This points to future studies into the dynamics of the thermal-piezoresistive feedback parameter, which has not been previously considered.

An auxiliary source of dissipation is responsible for the amplitude stabilization during repulsive contact. The piecewise modeling of the forced response in Fig. 1 reveals this dissipation pathway, since the predicted frequency upshift during contact would be much larger than the experimentally measured values in the absence of this dissipation. The Supplemental Material [74] addresses this point further, by showing the modeled amplitude and phase curves in the absence of contact dissipation. One possible origin for the auxiliary viscous damping and nonlinear damping is squeeze-film damping of the residual hydrogen gas molecules in the gap. The self-oscillation measurements on the repulsive-contactstabilized device in Fig. 4 provides additional evidence for the role of this auxiliary dissipation in stabilizing the amplitude during the repulsive contact, since increasing the feedback strength causes the steady-state oscillation frequency to decrease. If stiffening nonlinearity associated with the Hertzian contact of the asperities was the relevant amplitude stabilization nonlinearity, the oscillation frequency would increase with increasing feedback strength [20].

An interesting observation from these experiments is that to improve the oscillator frequency stability, the feedback strength for the nonlinear-damping-stabilized oscillator should be as large as possible while avoiding wall collisions, while the feedback strength for the repulsivecontact-stabilized oscillator should be as close to threshold as possible. If the feedback strength substantially exceeds threshold for the repulsive-contact-stabilized device, the surface asperities will more directly contact and perturb the oscillation frequency, as in Fig. 4(e). The repulsivecontact-stabilized oscillator appears to be less sensitive to fluctuations in oscillator properties near threshold than the nonlinear-damping-stabilized oscillator. The drift decreases with reducing feedback strength for both oscillators, but this results in a better frequency stability for only the repulsive-contact-stabilized oscillator because of its lower sensitivity to fluctuations in oscillator parameters near threshold. Eq. (21) provides one explanation for this difference: in the nonlinear-damping-stabilized oscillator, the vibration amplitude directly depends on feedback strength, while the amplitude in the repulsive-contact-stabilized oscillator is fixed by the gap size. So fluctuations in the feedback can more directly translate to fluctuations in the stability measures in the nonlinear-damping-stabilized oscillator than in the repulsivecontact-stabilized oscillator.
Measurements of the frequency stability in nonlineardamping-stabilized devices for time scales longer than those considered in Fig. 3 reveal that the resonance frequency drift accompanying the fatigue completely swamps other noise sources, and nonlinear-damping-stabilized oscillators stop functioning after 10 million to 100 million cycles. The repulsive-contact-stabilized devices, on the other hand, exhibited no apparent performance degradation after 1 billion cycles, other than wearing down the contacting asperities and reducing the corresponding frequency upshift during stabilization. The stability measures in Table I are an order-ofmagnitude better for the repulsive-contact-stabilized oscillator than the nonlinear-damping-stabilized oscillator for $\tau=1 \mathrm{~s}$. Because the internal feedback mechanism and the amplitude limiting mechanism are both localized in the engine beam during oscillations of the nonlinear-damping-stabilized oscillator, this seems to correspond to accelerated device degradation. For the repulsive-contact-stabilized oscillator, squeeze film damping in the electrode gaps serves as the amplitude limiting mechanism, so the engine beam is only responsible for the internal feedback. Spatially delocalizing the amplitude stabilization nonlinearity from the internal feedback in the device appears to improve long-term nonlinear oscillator performance.

The best achieved resonance frequency Allan deviation, $\sigma_{\omega_{0}}=3.4 \times 10^{-7}$ for the repulsive-contact-stabilized oscillator at an averaging time of $69 \mathrm{~ms}$, is comparable to the state of the art for thermal-piezoresistive oscillators, $\sigma_{\omega_{0}}=7.2 \times 10^{-8}$ at an averaging time of $22 \mathrm{~ms}$ [77], but is several orders of magnitude worse than the state of the art for all MEMS-based oscillators: $\sigma_{\omega_{0}}=9 \times 10^{-10}$ at an averaging time of $20 \mathrm{~s}$ [78]. The piezoresistive readout is not sensitive enough to detect the thermomechanical vibrations for our devices, so $\sigma_{\omega_{0}}$ can be improved in the white-noise-dominated regime $(\tau<10 \mathrm{~ms})$ by reducing the cross-sectional area of the piezoresistive element to increase the piezoresistive sensitivity $[63,79,80]$. Improving $\sigma_{\omega_{0}}$ in the drift-dominated regime $(\tau>10 \mathrm{~ms})$ would require further engineering of the amplitude limiting mechanism to eliminate the associated gradual degradation in oscillator properties, for either geometric nonlinear damping or repulsive contact. If the inherent drift in oscillator properties that accompanies thermal-piezoresistive pumping and the amplitude stabilization is minimized, then the drift due to temperature effects can be minimized with compensation [81]. Alternately, the drift in oscillator properties that accompanies thermal-piezoresistive pumping can potentially be bypassed by using a different internal feedback mechanism, such as optical pumping [2], to sustain oscillations.

The nonlinearities that we explore in this work are generalizable to oscillators sustained via photon-cavity [3], magnon-cavity [8], or phonon-cavity [10] mechanics, as well as external feedback oscillators [1,21]. Our measurements and simulations reveal the interesting dynamics that can occur in MEM/NEM oscillators that are stabilized by geometric nonlinear damping and repulsive contact, and considers the associated implications for nonlinear oscillator performance. In order to improve the utility of geometric nonlinear damping within MEM/NEM oscillators; further work is required to engineer the nonlinear damping in the high stress regions of the resonator to stabilize the amplitude without the signatures 
of fatigue that we observe here. Because of the larger vibration amplitude, oscillators stabilized with internal nonlinearity, such as the amplitude stabilization mechanisms that we delineate, offer an inherent thermomechanical resonance frequency stability advantage over linear oscillators, which translates to overall stability improvements if the displacement readout is thermomechanical-noise-limited [82], accompanying amplitude-frequency noise conversion is mitigated [16], and auxiliary frequency fluctuations are not present [79]. Because nonlinear damping is essential for the amplitude stabilization in nonlinear oscillators, the frequency fluctuations that are predicted to accompany the nonlinear damping should also be identified and minimized [55].

\section{CONCLUSION AND OUTLOOK}

We delineate two kinds of amplitude stabilization mechanisms in hybrid vibrational systems sustained via the coupling between electrons and mechanical vibrations: geometric nonlinear damping and repulsive contact. These two nonlinearities are generalizable to micromechanical oscillators sustained by coupling to a wide variety of internal degrees of freedom, such as photons, magnons, or auxiliary mechanical modes. These amplitude stabilization mechanisms can coexist in the same device and their interplay and corresponding frequency stability can be tuned in-situ by adjusting the feedback strength. An auxiliary source of viscous dissipation and nonlinear dissipation accompanies the repulsive contact, potentially arising from squeeze film damping. We anticipate that significant improvements to frequency stability beyond what we report here can be achieved by further engineering the geometric nonlinear damping and the repulsive contact amplitude stabilization mechanisms.

\section{ACKNOWLEDGMENTS}

J.M.L.M. is grateful for the helpful discussions with Alp Sipahigil and Steven Shaw. This work is supported by the National Science Foundation Collaborative Research Program under Award No. CMMI-1662464. Fabrication was performed in the nano@Stanford labs, which are supported by the National Science Foundation (NSF) as part of the National Nanotechnology Coordinated Infrastructure under Award No. ECCS-1542152, with support from the Defense Advanced Research Projects Agency Precise Robust Inertial Guidance for Munitions (PRIGM) Program, managed by Ron Polcawich and Robert Lutwak. J.M.L.M. was partially supported by the National Defense Science and Engineering Graduate (NDSEG) Fellowship and the E.K. Potter Stanford Graduate Fellowship.

\section{APPENDIX A: MEASUREMENTS}

The piezoresistive readout measures the vibrations via a change in electrical resistance in the engine beam during oscillations. Our differential piezoresistive buffer eliminates any common-mode noise between the voltage output from the two anchors. A four-probe resistive setup is employed, whereby the direct current flows through the resonator via a pair of electrical contacts through the encapsulation to the anchors in the device layer, and a second pair of electrical contacts to the anchors are used to read out the voltages induced by the changing electrical resistance. This bypasses the significant parasitic resistance in the electrical contacts during the resistive measurement and improves the piezoresistive signal.

The capacitive readout measures the vibrations via the changing capacitive gap width between the resonator and the transduction electrode in the presence of a static voltage difference between the two conductors. The transimpedance amplifier is designed to measure the induced motional current in the transduction electrode at the self-oscillation amplitudes, without distortion.

The output responsivities, in units of $\mathrm{V} / \mathrm{m}$, are obtained for the piezoresistive and capacitive readouts directly from the self-sustained oscillations during contact with the stabilization electrode and the a priori gap size. This calibration is simpler and more accurate at large amplitudes than the thermomechanical-noise-based calibration procedure [82], because of the significant capacitive nonlinearity for vibrations comparable to the gap size and piezoresistive readout inaccuracy when extrapolating to the large self-oscillation currents. The Supplemental Material [74] compares the displacement calibration results based on the contact calibration and the thermomechanical noise calibration for the devices presented in the manuscript.

The forced responses presented in Fig. 1 are obtained using a Zurich Instruments (ZI) lock-in amplifier. The ZI output waveform generator is connected to the transduction electrode, and the output from the piezoresistive readout is connected to the ZI input for demodulation. During the phase-locked loop measurements, the ZI establishes a proportional-integral-derivative (PID) loop on the phase difference between the output drive voltage and input piezoresistive signal at the same frequency. Near resonance, the phase difference between the ZI drive output and the output from the piezoresistive readout depends sensitively on the ZI drive frequency. By sweeping the phase set-point from $0^{\circ}$ to $-180^{\circ}$ in $100 \mathrm{mHz}$ steps, the entire nonlinear resonator response including the stable and unstable branches can be measured. Open-loop operation in the nonlinear regime measures multi-valued frequency-amplitude and frequencyphase curves, which include stable and unstable branches, and is unable to access the unstable branches of the response. Closed-loop operation, as is used to obtain the forced responses in Fig. 1, measures the phase-amplitude and phasefrequency curves, which are always single-valued [83]. The PID loop parameters were determined using the ZI phaselocked loop advisor by inputting the resonance frequency and quality factor of the mode. See Sec. III of the Supplemental Material [74] for more information about the tested device in Fig. 1 and the comparison between the measured and modeled forced response.

For the self-oscillation measurements presented in Figs. 2 4 , the output voltages from the piezoresistive and capacitive readouts are measured with a dual-channel Alazar ATS9360$4 \mathrm{G}$ digital acquisition board, at a $10 \mathrm{MHz}$ sampling rate. The single-period resonance frequencies are extracted from the time series of the self-oscillation measurements, by calculating each period $T$ from the interpolated zero-crossings and employing the relation $\omega_{0}=2 \pi / T$. The positive and negative 

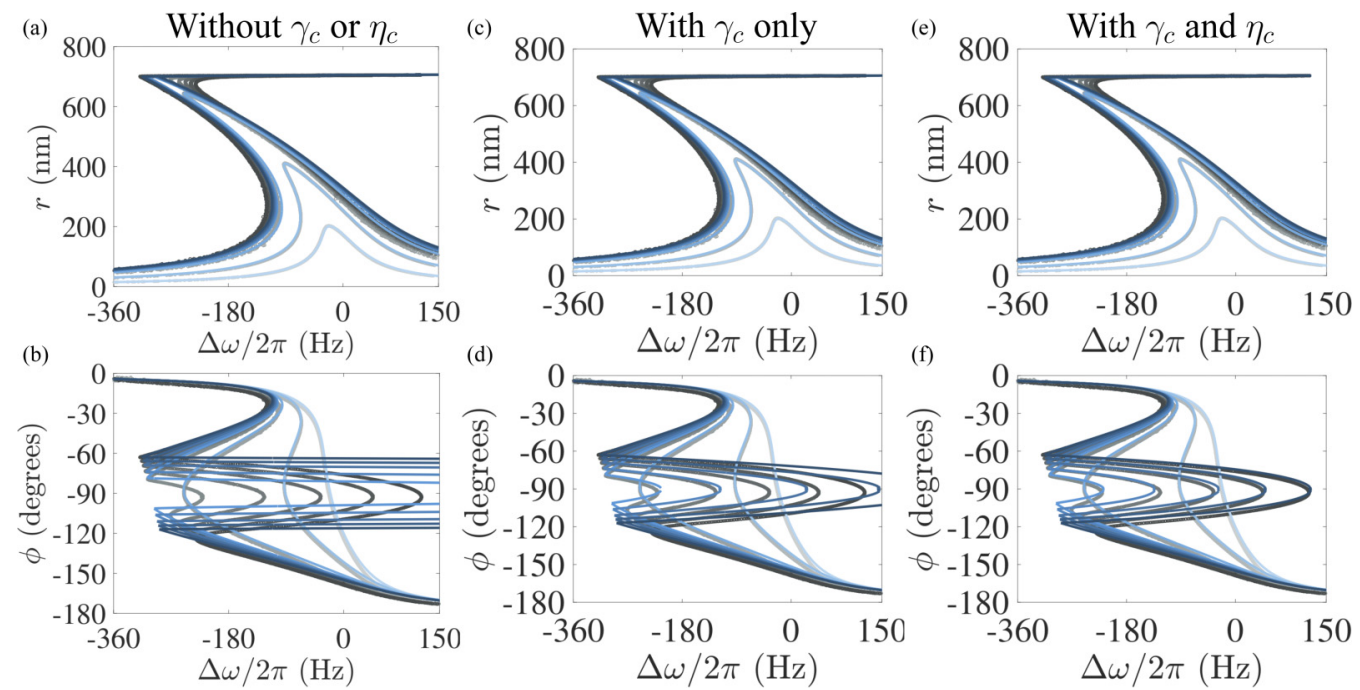

FIG. 5. Measuring the contact dissipation during repulsive contact. [(a),(b)] The measured amplitude and phase curves (gray circles) with the overlaid simulations (blue curves), including neither contact viscous damping nor contact nonlinear damping. [(c),(d)] The measured amplitude and phase curves with the overlaid simulations, including contact viscous damping but not including contact nonlinear damping. [(e),(f)] The measured amplitude and phase curves with the overlaid simulations, including both contact viscous damping and contact nonlinear damping.

values of the amplitude envelope in each vibration cycle are extracted from the maximum and minimum measured points between zero-crossings, respectively. To plot the statistics of the amplitude and resonance frequency in Figs. 3(a), 3(b), 3(e), 3(f), 3(i), 3(j), and 4(b), the values from each period are first averaged into $\tau=500 \mu \mathrm{s}$ bins, and then 100 of these bins are used to compute the mean and standard deviation in $50 \mathrm{~ms}$ time intervals. The self-oscillation frequency during the long-time measurements in Fig. 4(e) is recorded with an Agilent 53132A universal counter at a sampling rate of roughly $\omega_{s} / 2 \pi=1 \mathrm{~Hz}$ immediately following the initial ring-up measurements shown in Fig. 4(a).

For $N$ total measurements of resonance frequency, the resonance frequency Allan deviation is calculated for a given averaging time $\tau$ as [79]

$$
\sigma_{\omega_{0}}(\tau)=\sqrt{\frac{1}{2\left(\frac{N}{m}-1\right)} \sum_{i=1}^{\frac{N}{m}-1}\left(\frac{\bar{\omega}_{0, i+1}-\bar{\omega}_{0, i}}{\omega_{0}}\right)^{2}},
$$

where $m=\tau \omega_{s} / 2 \pi$ is the number of samples in each averaged resonance frequency $\bar{\omega}_{0, i} / 2 \pi$ and $\omega_{0} / 2 \pi$ is the reference (e.g., initial) resonance frequency. For short averaging times, there are fewer samples averaged into each $\bar{\omega}_{0, i}$ than when the averaging times are longer.

The amplitude Allan deviation is similarly computed as

$$
\sigma_{r}(\tau)=\sqrt{\frac{1}{2\left(\frac{N}{m}-1\right)} \sum_{i=1}^{\frac{N}{m}-1}\left(\frac{\bar{r}_{i+1}-\bar{r}_{i}}{r}\right)^{2}},
$$

where $\bar{r}_{i}$ is the averaged amplitude in the $i$ th time bin and $r$ is the reference amplitude, corresponding to the initial steadystate amplitude following the transient ring-up.

For the Allan deviations corresponding to the zero-crossing resonance frequency and envelope amplitude in Figs. 3(c), 3(d), 3(g), 3(h), 3(k), 3(1), 4(c), and 4(d), the sampling rate is roughly equal to the resonance frequency: $\omega_{s}=\omega_{0}$. For the resonance frequency Allan deviation curves shown in Fig. 4(f), the sampling rate corresponds to the frequency counter measurement rate, and is roughly $1 \mathrm{~Hz}$.

\section{APPENDIX B: MEASURING THE CONTACT VISCOUS/NONLINEAR DISSIPATION}

Figures 5(a) and 5(b) show the experimentally measured forced oscillation curves for the long support beam $(L=50 \mu \mathrm{m})$ narrow gap $(g=700 \mathrm{~nm})$ device, with the corresponding nonlinear model in the absence of the contact viscous damping and piecewise nonlinear damping. The measurements are the same as in Figs. 1(d) and 1(e). The bias voltage is set to $V_{b}=10 \mathrm{~V}$, and a piezoresistive sensing current $I_{\mathrm{dc}}=1 \mathrm{~mA}$ is used. The drive amplitude parameter is chosen for each simulated curve so that the discontinuity in the phase slope for the experiment and simulation near $-75^{\circ}$ occurs at the same phase. The contact stiffness parameter is chosen so that the slope of the amplitude increase to increasing drive strength agrees between the experiments and simulations. The simulated phase curves join together at a much larger frequency offset from resonance than the experimental value, which suggests that conservative stiffening nonlinearity alone is not solely responsible for the amplitude stabilization during repulsive contact.

Figures 5(c) and 5(d) show the experimental curves with overlaid simulations, which includes the contribution from contact viscous damping but not contact nonlinear damping. The inclusion of the piecewise viscous damping enables the simulated curves to agree with the experimental curves during contact at low amplitudes, but overestimates the frequency offset that the phase curves join together at for larger drive amplitudes; piecewise viscous damping alone is not sufficient to describe the dissipative contact forces. 


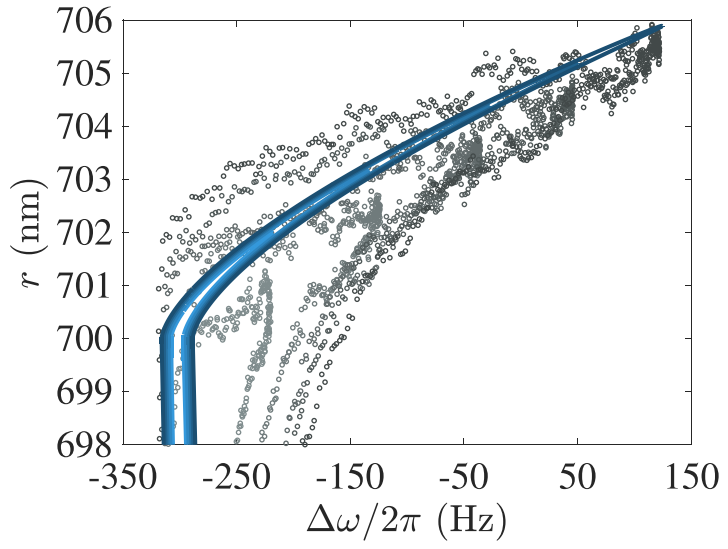

FIG. 6. A close-up view of the measured amplitude-frequency curves (gray circles) and the overlaid simulations (blue curves), with contact viscous damping and contact nonlinear damping.

Figures 5(e) and 5(f) show the experimental curves with overlaid simulations, which includes the contribution from both contact viscous damping and contact nonlinear damping. The simulated curves are identical to those presented in Figs. 1(f) and 1(g). The inclusion of the piecewise nonlinear damping significantly improves the agreement between the simulations and experiments for the largest vibration amplitudes. The corresponding close-up view of the amplitude-frequency curves with the overlaid model is shown in Fig. 6.

Comparing the lumped nonlinear model to the measurements indicates that piecewise linear stiffness, piecewise viscous damping, and piecewise nonlinear damping capture the essential dynamics of the device during repulsive contact, including the slope of the amplitude-frequency curves and the frequencies that the stable and unstable amplitude branches join together at. The values of the nonlinear parameters used for this model are summarized in Table II. The nonlinear model does not completely capture the nonlinear forces during the repulsive contact: the model underestimates the frequency offset at which the the phase slope experiences a second discontinuity in Fig. 5(f), near $-120^{\circ}$, as well as the phase slope near that discontinuity. Additionally, the amplitude model in Fig. 6 underestimates the width of the resonance peak in the stiffening region at amplitudes slightly below and above sidewall contact. The error between the experimental and modeled amplitude curves in the repulsive contact region are within 1-2 nm. A deeper study of the resonator-sidewall interaction forces may enable an additional level of refinement to our nonlinear model. However, the model that we present here is sufficient for revealing the additional viscous damping and nonlinear damping that are at play during repulsive contact.

\section{APPENDIX C: NUMERICAL SOLUTION OF THE GOVERNING EQUATIONS}

To plot the amplitude-frequency and phase-frequency curves from the nonlinear piecewise model in Figs. 1, 5, and 6, we numerically solve Eqs. (4) and (5). We define a rootfinding function by subtracting $r$ from both sides of Eq. (4). The zeros of this function correspond to the physical amplitude solutions at a given drive amplitude and frequency. For each drive force, we first compute the entire linear response over the frequency range around resonance by setting $I_{\text {even }}$ and $I_{\text {odd }}$ to zero within Eq. (4). The amplitude at resonance is used to choose the upper bound for a coarse open search on the root-finding function [84]. The coarse zero crossings in the open search are subsequently refined using the false-position method, which ultimately outputs one or more $r, I_{\text {even }}$, and $I_{\text {odd }}$ values (corresponding to each branch of the nonlinear response) at a given $\omega$ and $f$. The corresponding phase values for each branch are computed using the outputted $I_{\text {even }}$ and $I_{\text {odd }}$ values from the root finder. Each branch of the amplitude and phase response at a given drive amplitude is stored into its own vector. All of the stored branches are shown in Figs. 1(f) and $1(\mathrm{~g})$

\section{APPENDIX D: DERIVATION OF THE NONLINEAR MODEL}

\section{Nonlinearities arising from the electrostatics}

The bias voltage difference between the microcantilever and the adjacent transduction electrode stores electrostatic energy $U_{e}$ in the parallel-plate capacitance of the gap:

$$
U_{e}(x)=-\frac{\epsilon_{0} A}{2(g-x)},
$$

where $\epsilon_{0}=8.854 \times 10^{-12} \mathrm{~F} \mathrm{~m}^{-1}$ is the vacuum permittivity, $A$ is the electrode area, $g$ is the capacitive gap size in static equilibrium, and $x$ is the resonator displacement away from equilibrium. The displacement-dependence of the electrostatic energy gives rise to a mass-normalized attractive force:

$$
f_{e}(x)=\frac{c_{e}}{(g-x)^{2}},
$$

where the lumped-mass-normalized electrostatic constant is given by

$$
c_{e}=\frac{\epsilon_{0} A V_{b}^{2}}{2 m} .
$$

Performing a Taylor series expansion on Eq. (D2) up to fifth-order and discarding the nonresonant forcing terms,

TABLE II. A delineation of the nonlinear lumped element model values for the device measured and simulated in Figs. 1, 5, and 6. $\omega_{0}$ is the angular resonance frequency, $Q$ is the quality factor, $\gamma$ is the linewidth, $g$ is the gap size, $\eta$ is the nonlinear damping parameter, $\omega_{c}$ is the contact linear stiffness parameter, $\gamma_{c}$ is the contact viscous damping parameter, and $\eta_{c}$ is the contact nonlinear damping parameter. $\eta$ is set to zero in the model for this device.

\begin{tabular}{lcccccccc}
\hline \hline$\omega_{0} / 2 \pi(\mathrm{kHz})$ & $Q$ & $\gamma\left(\mathrm{rad} \mathrm{s}^{-1}\right)$ & $g(\mathrm{~nm})$ & $\alpha\left(\mathrm{rad}^{2} \mathrm{~m}^{-2} \mathrm{~s}^{-2}\right)$ & $\beta\left(\mathrm{rad}^{2} \mathrm{~m}^{-4} \mathrm{~s}^{-2}\right)$ & $\omega_{c}^{2}\left(\mathrm{rad}^{2} \mathrm{~s}^{-2}\right)$ & $\gamma_{c}\left(\mathrm{rad} \mathrm{s}^{-1}\right)$ & $\eta_{c}\left(\mathrm{rad} \mathrm{m}^{-2} \mathrm{~s}^{-1}\right)$ \\
\hline 278.395 & 5200 & 336.4 & 700 & $-1.48 \times 10^{22}$ & $-8.11 \times 10^{33}$ & $2.08 \times 10^{13}$ & $5.93 \times 10^{4}$ & $2.91 \times 10^{21}$ \\
\hline \hline
\end{tabular}


we obtain

$$
f_{e}(x)=2 c_{e} g^{-3} x+4 c_{e} g^{-5} x^{3}+6 c_{e} g^{-7} x^{5} .
$$

The corresponding Duffing (Kerr) parameter and fifth-order nonlinear parameter, arising from electrostatics, are, respectively,

$$
\begin{aligned}
\alpha_{e} & =-\frac{2 \epsilon_{0} A V_{b}^{2}}{m g^{5}}, \\
\beta_{e} & =-\frac{3 \epsilon_{0} A V_{b}^{2}}{m g^{7}} .
\end{aligned}
$$

Using a bias voltage $V_{b}=10 \mathrm{~V}$, an electrode area of $A=$ $1.44 \times 10^{-8} \mathrm{~m}^{2}$, a gap size $g=700 \mathrm{~nm}$, and a lumped mass $m=4.809 \times 10^{-9} \mathrm{~kg}$ as for the device in the forced vibration experiments, we compute $\alpha_{e}=-3.16 \times 10^{22} \mathrm{rad} \mathrm{m}^{-2} \mathrm{~s}^{-2}$ and $\beta_{e}=-9.66 \times 10^{34} \mathrm{rad} \mathrm{m}^{-4} \mathrm{~s}^{-2}$. These estimated values are reasonably close to measured parameter values in Table II.

\section{Nonlinearities arising from the van der Waals force}

The attractive van der Waals force between an asperity on the resonator or electrode sidewall and the opposing surface, normalized by the resonator lumped mass, is given by

$$
f_{v}=\frac{c_{v}}{(g-x)^{2}},
$$

where the lumped-mass-normalized van der Waals constant is given by

$$
c_{v}=\frac{A_{H} R}{6 m},
$$

where $A_{H}$ is the Hamaker constant and $R$ is the asperity radius. The van der Waals force in Eq. (D7) has the same functional form as the electrostatic force in Eq. (D2), and thus the power series expansion has the same form:

$$
f_{v}(x)=2 c_{v} g^{-3} x+4 c_{v} g^{-5} x^{3}+6 c_{v} g^{-7} x^{5} .
$$

The corresponding Duffing (Kerr) parameter and fifth-order nonlinear parameter, arising from the van der Waals force, are respectively,

$$
\begin{gathered}
\alpha_{v}=-\frac{2 A_{H} R}{3 m g^{5}}, \\
\beta_{v}=-\frac{A_{H} R}{m g^{7}} .
\end{gathered}
$$

Using a Hamaker constant $A_{H}=2 \times 10^{-18} \mathrm{~J}$ and an asperity radius $R=10 \mathrm{~nm}$, reasonable for scanning probe microscopy experiments [62], we estimate $\alpha_{v}=-1.65 \times 10^{12} \mathrm{rad} \mathrm{m}^{-2} \mathrm{~s}^{-2}$ and $\beta_{e}=-5.05 \times 10^{24} \mathrm{rad} \mathrm{m}^{-4} \mathrm{~s}^{-2}$. It is clear that the van der Waals contribution to the nonlinear model is negligible compared to the electrostatic contribution for the electrically biased device.

\section{Expansion of the Hertzian contact force}

The attractive and repulsive forces between an asperity and an adjacent surface can be described by the Derjaguin, Muller, Toporov, and Maugis (DMT-M) theory. This corresponds to a piecewise nonlinear tip-sidewall force, which includes both an attractive van der Waals force and a repulsive Hertzian contact force [62]:

$$
f_{t s}= \begin{cases}-c_{v}(x-g)^{-2}, & x<g, \\ -c_{v}(x-g)^{-2}+c_{H}(x-g)^{3 / 2}, & x \geqslant g,\end{cases}
$$

where $c_{v}$ is the van der Waals force constant defined in the previous section. We define the Hertzian force constant as

$$
c_{H}=\frac{4 E \sqrt{R}}{3 m},
$$

where $E$ is the elastic modulus of the asperity and the sidewall. The Hertzian contact force can be expanded in a power series about $x=g$ by assuming that the resonator first contacts an asperity at $x=g-R$, and discarding the contact force for $x<g$. This approximation yields

$$
f_{H}= \begin{cases}0, & x<g, \\ -\frac{3}{2} c_{H} R^{1 / 2}(x-g)-\frac{1}{16} c_{H} R^{-3 / 2}(x-g)^{3}, & x \geqslant g .\end{cases}
$$

The corresponding linear contact parameter is thus:

$$
\omega_{c}^{2}=\frac{2 E R}{m} .
$$

Using a silicon elastic modulus $E=130 \mathrm{GPa}$ and an asperity radius of $R=10 \mathrm{~nm}$, we compute $\omega_{c}^{2}=5.41 \times 10^{11} \mathrm{rad}^{2} \mathrm{~s}^{-2}$ for the linear contact parameter. The actual linear contact parameter is expected to be larger than this approximate value if multiple asperities come into contact at $x \approx g$, as the measured value for $\omega_{c}^{2}$ in Table II confirms.

\section{Computing the Fourier coefficients}

The Fourier components $I_{\text {even }}$ and $I_{\text {odd }}$ in Eqs. (8) and (9) are evaluated by pre-multiplying the nonlinear force by the appropriate sinusoid and integrating the function over a single vibration period:

$$
\begin{aligned}
& I_{\text {even }}(\omega, r)=\frac{1}{\pi \omega_{0}^{2} r} \int_{0}^{2 \pi} f_{\mathrm{NL}}(\omega, r, \tau) \sin (\tau) d \tau, \\
& I_{\mathrm{odd}}(\omega, r)=\frac{1}{\pi \omega_{0}^{2} r} \int_{0}^{2 \pi} f_{\mathrm{NL}}(\omega, r, \tau) \cos (\tau) d \tau,
\end{aligned}
$$

where $f_{\mathrm{NL}}$ is the mass-normalized nonlinear force, $\omega$ is the angular drive frequency, $\omega_{0}$ is the angular resonance frequency, $r$ is the vibration amplitude, and $\tau=\omega t+\phi$ is the slow time. The contribution of the nonlinear forces to the response is captured in the following Fourier coefficient integrals:

$$
\begin{aligned}
I_{\text {even }, c}(\omega, r)= & \frac{1}{\pi \omega_{0}^{2} r} \int_{0}^{\tau_{1}} f_{c}(\omega, r, \tau) \sin (\tau) d \tau \\
& +\frac{1}{\pi \omega_{0}^{2} r} \int_{2 \pi-\tau_{1}}^{2 \pi} f_{c}(\omega, r, \tau) \sin (\tau) d \tau, \\
I_{\text {odd }, c}(\omega, r)= & \frac{1}{\pi \omega_{0}^{2} r} \int_{0}^{\tau_{1}} f_{c}(\omega, r, \tau) \cos (\tau) d \tau \\
& +\frac{1}{\pi \omega_{0}^{2} r} \int_{2 \pi-\tau_{1}}^{2 \pi} f_{c}(\omega, r, \tau) \cos (\tau) d \tau,
\end{aligned}
$$

where $f_{c}$ is the mass-normalized contact force and $g$ is the gap size. $\tau_{1}=\operatorname{acos}(g / r)$ characterizes the fraction of the oscilla- 
tion period spent in contact with the sidewall. These integrals assume that the resonator contacts a single surface at $x=g$ during oscillations. We make this assumption since the direct current through the engine beam causes a static shift in the resonator proof mass towards the stabilization electrode. If the resonator simultaneously contacts both adjacent electrodes during self-oscillations, at $x= \pm g$, then Eqs. (10), (11), and (12) will be premultiplied by 2 , and the corresponding nonlinear contact parameters will assume half the value presented in Table II.
[1] X. L. Feng, C. J. White, A. Hajimiri, and M. L. Roukes, A self-sustaining ultrahigh-frequency nanoelectromechanical oscillator, Nat. Nanotechnol. 3, 342 (2008).

[2] M. Hossein-Zadeh, H. Rokhsari, A. Hajimiri, and K. J. Vahala, Characterization of a radiation-pressure-driven micromechanical oscillator, Phys. Rev. A 74, 023813 (2006).

[3] A. H. Safavi-Naeini, D. Van Thourhout, R. Baets, and R. Van Laer, Controlling phonons and photons at the wavelength scale: Integrated photonics meets integrated phononics, Optica 6, 213 (2019).

[4] I. Shomroni, A. Youssefi, N. Sauerwein, L. Qiu, P. Seidler, D. Malz, A. Nunnenkamp, and T. J. Kippenberg, Two-Tone Optomechanical Instability and its Fundamental Implications for Backaction-Evading Measurements, Phys. Rev. X 9, 041022 (2019).

[5] G. A. Peterson, S. Kotler, F. Lecocq, K. Cicak, X. Y. Jin, R. W. Simmonds, J. Aumentado, and J. D. Teufel, Ultrastrong Parametric Coupling between a Superconducting Cavity and a Mechanical Resonator, Phys. Rev. Lett. 123, 247701 (2019).

[6] T. Barois, S. Perisanu, P. Vincent, S. T. Purcell, and A. Ayari, Role of fluctuations and nonlinearities on field emission nanomechanical self-oscillators, Phys. Rev. B 88, 195428 (2013).

[7] C. Urgell, W. Yang, S. De Bonis, C. Samanta, M. J. Esplandiu, Q. Dong, Y. Jin, and A. Bachtold, Cooling and self-oscillation in a nanotube electromechanical resonator, Nat. Phys. 16, 32 (2020).

[8] X. Zhang, C.-L. Zou, L. Jiang, and H. X. Tang, Cavity magnomechanics, Sci. Adv. 2, e1501286 (2016).

[9] D. Lachance-Quirion, Y. Tabuchi, A. Gloppe, K. Usami, and Y. Nakamura, Hybrid quantum systems based on magnonics, Appl. Phys. Express 12, 070101 (2019).

[10] I. Mahboob, K. Nishiguchi, H. Okamoto, and H. Yamaguchi, Phonon-cavity electromechanics, Nat. Phys. 8, 387 (2012).

[11] M. Serra-Garcia, A. Foehr, M. Molerón, J. Lydon, C. Chong, and C. Daraio, Mechanical Autonomous Stochastic Heat Engine, Phys. Rev. Lett. 117, 010602 (2016).

[12] X. Zhou, C. Zhao, D. Xiao, J. Sun, G. Sobreviela, D. D. Gerrard, Y. Chen, I. Flader, T. W. Kenny, X. Wu et al., Dynamic modulation of modal coupling in microelectromechanical gyroscopic ring resonators, Nat. Commun. 10, 4980 (2019).

[13] J. M. L. Miller, A. Ansari, D. B. Heinz, Y. Chen, I. B. Flader, D. D. Shin, L. G. Villanueva, and T. W. Kenny, Effective quality factor tuning mechanisms in micromechanical resonators, Appl. Phys. Rev. 5, 041307 (2018).

[14] D. S. Greywall, B. Yurke, P. A. Busch, A. N. Pargellis, and R. L. Willett, Evading Amplifier Noise in Nonlinear Oscillators, Phys. Rev. Lett. 72, 2992 (1994).

[15] L. G. Villanueva, E. Kenig, R. B. Karabalin, M. H. Matheny, R. Lifshitz, M. C. Cross, and M. L. Roukes, Surpassing Funda- mental Limits of Oscillators using Nonlinear Resonators, Phys. Rev. Lett. 110, 177208 (2013).

[16] L. Huang, S. M. Soskin, I. A. Khovanov, R. Mannella, K. Ninios, and H. B. Chan, Frequency stabilization and noiseinduced spectral narrowing in resonators with zero dispersion, Nat. Commun. 10, 3930 (2019).

[17] D. Antonio, D. H. Zanette, and D. López, Frequency stabilization in nonlinear micromechanical oscillators, Nat. Commun. 3, 1 (2012).

[18] F. Sun, X. Dong, J. Zou, M. I. Dykman, and H. B. Chan, Correlated anomalous phase diffusion of coupled phononic modes in a sideband-driven resonator, Nat. Commun. 7, 12694 (2016).

[19] J. Güttinger, A. Noury, P. Weber, A. M. Eriksson, C. Lagoin, J. Moser, C. Eichler, A. Wallraff, A. Isacsson, and A. Bachtold, Energy-dependent path of dissipation in nanomechanical resonators, Nat. Nanotechnol. 12, 631 (2017).

[20] C. Chen, D. H. Zanette, D. A. Czaplewski, S. Shaw, and D. López, Direct observation of coherent energy transfer in nonlinear micromechanical oscillators, Nat. Commun. 8, 15523 (2017).

[21] C. Chen, D. H. Zanette, J. R. Guest, D. A. Czaplewski, and D. López, Self-Sustained Micromechanical Oscillator with Linear Feedback, Phys. Rev. Lett. 117, 017203 (2016).

[22] A. D. O'Connell, M. Hofheinz, M. Ansmann, R. C. Bialczak, M. Lenander, E. Lucero, M. Neeley, D. Sank, H. Wang, M. Weides et al., Quantum ground state and single-phonon control of a mechanical resonator, Nature (London) 464, 697 (2010).

[23] J. D. Teufel, T. Donner, D. Li, J. W. Harlow, M. S. Allman, K. Cicak, A. J. Sirois, J. D. Whittaker, K. W. Lehnert, and R. W. Simmonds, Sideband cooling of micromechanical motion to the quantum ground state, Nature (London) 475, 359 (2011).

[24] J. Chan, T. P. M. Alegre, A. H. Safavi-Naeini, J. T. Hill, A. Krause, S. Gröblacher, M. Aspelmeyer, and O. Painter, Laser cooling of a nanomechanical oscillator into its quantum ground state, Nature (London) 478, 89 (2011).

[25] R. Riedinger, A. Wallucks, I. Marinković, C. Löschnauer, M. Aspelmeyer, S. Hong, and S. Gröblacher, Remote quantum entanglement between two micromechanical oscillators, Nature (London) 556, 473 (2018).

[26] K. J. Satzinger, Y. P. Zhong, H.-S. Chang, G. A. Peairs, A. Bienfait, M.-H. Chou, A. Y. Cleland, C. R. Conner, É. Dumur, J. Grebel et al., Quantum control of surface acoustic-wave phonons, Nature (London) 563, 661 (2018).

[27] P. Arrangoiz-Arriola, E. A. Wollack, Z. Wang, M. Pechal, W. Jiang, T. P. McKenna, J. D. Witmer, R. Van Laer, and A. H. Safavi-Naeini, Resolving the energy levels of a nanomechanical oscillator, Nature (London) 571, 537 (2019).

[28] Y. Tsaturyan, A. Barg, E. S. Polzik, and A. Schliesser, U1tracoherent nanomechanical resonators via soft clamping and dissipation dilution, Nat. Nanotechnol. 12, 776 (2017). 
[29] A. H. Ghadimi, S. A. Fedorov, N. J. Engelsen, M. J. Bereyhi, R. Schilling, D. J. Wilson, and T. J. Kippenberg, Elastic strain engineering for ultralow mechanical dissipation, Science $\mathbf{3 6 0}$, 764 (2018).

[30] G. S. MacCabe, H. Ren, J. Luo, J. D. Cohen, H. Zhou, A. Sipahigil, M. Mirhosseini, and O. Painter, Nano-acoustic resonator with ultralong phonon lifetime, Science 370, 840 (2020).

[31] T. Ramos, V. Sudhir, K. Stannigel, P. Zoller, and T. J. Kippenberg, Nonlinear Quantum Optomechanics Via Individual Intrinsic Two-Level Defects, Phys. Rev. Lett. 110, 193602 (2013).

[32] F. Pistolesi, A. N. Cleland, and A. Bachtold, Proposal for A Nanomechanical Qubit, Phys. Rev. X 11, 031027 (2021).

[33] S. Chiavazzo, A. S. Sørensen, O. Kyriienko, and L. Dellantonio, Quantum manipulation of a two-level mechanical system, arXiv:2101.01750.

[34] J. Suh, M. D. LaHaye, P. M. Echternach, K. C. Schwab, and M. L. Roukes, Parametric amplification and back-action noise squeezing by a qubit-coupled nanoresonator, Nano Lett. 10, 3990 (2010).

[35] I. Mahboob, N. Perrissin, K. Nishiguchi, D. Hatanaka, Y. Okazaki, A. Fujiwara, and H. Yamaguchi, Dispersive and dissipative coupling in a micromechanical resonator embedded with a nanomechanical resonator, Nano Lett. 15, 2312 (2015).

[36] A. N. Cleland, Foundations of Nanomechanics: From SolidState Theory to Device Applications (Springer-Verlag, Berlin, 2013).

[37] M. H. Matheny, L. G. Villanueva, R. B. Karabalin, J. E. Sader, and M. L. Roukes, Nonlinear mode-coupling in nanomechanical systems, Nano Lett. 13, 1622 (2013).

[38] P. M. Polunin, Y. Yang, M. I. Dykman, T. W. Kenny, and S. W. Shaw, Characterization of MEMS resonator nonlinearities using the ringdown response, J. Microelectromech. Syst. 25, 297 (2016).

[39] F. Alijani, M. Amabili, P. Balasubramanian, S. Carra, G. Ferrari, and R. Garziera, Damping for large-amplitude vibrations of plates and curved panels, part 1: Modeling and experiments, Int. J. Non-linear Mech. 85, 23 (2016).

[40] D. Davidovikj, F. Alijani, S. J. Cartamil-Bueno, H. S. J. van der Zant, M. Amabili, and P. G. Steeneken, Nonlinear dynamic characterization of two-dimensional materials, Nat. Commun. 8, 1 (2017).

[41] E. Collin, Y. M. Bunkov, and H. Godfrin, Addressing geometric nonlinearities with cantilever microelectromechanical systems: Beyond the Duffing model, Phys. Rev. B 82, 235416 (2010).

[42] L. G. Villanueva, R. B. Karabalin, M. H. Matheny, D. Chi, J. E. Sader, and M. L. Roukes, Nonlinearity in nanomechanical cantilevers, Phys. Rev. B 87, 024304 (2013).

[43] V. Kaajakari, T. Mattila, A. Oja, and H. Seppa, Nonlinear limits for single-crystal silicon microresonators, J. Microelectromech. Syst. 13, 715 (2004).

[44] Y. Yang, E. J. Ng, P. M. Polunin, Y. Chen, I. B. Flader, S. W. Shaw, M. I. Dykman, and T. W. Kenny, Nonlinearity of degenerately doped bulk-mode silicon MEMS resonators, J. Microelectromech. Syst. 25, 859 (2016).

[45] R. Lifshitz and M. C. Cross, Nonlinear Dynamics of Nanomechanical Resonators (Wiley, Weinheim, 2008), Vol. 1, pp. 1-52.

[46] M. Agarwal, S. A. Chandorkar, H. Mehta, R. N. Candler, B. Kim, M. A. Hopcroft, R. Melamud, C. M. Jha, G. Bahl, G.
Yama et al., A study of electrostatic force nonlinearities in resonant microstructures, Appl. Phys. Lett. 92, 104106 (2008).

[47] J. M. L. Miller, D. D. Shin, H.-K. Kwon, S. W. Shaw, and T. W. Kenny, Phase Control of Self-Excited Parametric Resonators, Phys. Rev. Applied 12, 044053 (2019).

[48] S. Zaitsev, O. Shtempluck, E. Buks, and O. Gottlieb, Nonlinear damping in a micromechanical oscillator, Nonlinear Dyn. 67, 859 (2012).

[49] B. Jeong, H. Cho, M.-F. Yu, A. F. Vakakis, D. M. McFarland, and L. A. Bergman, Modeling and measurement of geometrically nonlinear damping in a microcantilever-nanotube system, ACS Nano 7, 8547 (2013).

[50] A. L. Alter, I. B. Flader, Y. Chen, D. D. Shin, and T. W. Kenny, Nonlinear dissipation in epitaxial SCS and polysilicon MEMS driven at large amplitudes, J. Microelectromech. Syst. 29, 1118 (2020).

[51] A. Eichler, J. Moser, J. Chaste, M. Zdrojek, I. Wilson-Rae, and A. Bachtold, Nonlinear damping in mechanical resonators made from carbon nanotubes and graphene, Nat. Nanotechnol. 6, 339 (2011).

[52] A. Keşkekler, O. Shoshani, M. Lee, H. S. van der Zant, P. G. Steeneken, and F. Alijani, Tuning nonlinear damping in graphene nanoresonators by parametric-direct internal resonance, Nat. Commun. 12, 1099 (2021).

[53] Z. Nourmohammadi, S. Mukherjee, S. Joshi, J. Song, and S. Vengallatore, Methods for atomistic simulations of linear and nonlinear damping in nanomechanical resonators, J. Microelectromech. Syst. 24, 1462 (2015).

[54] S. J. Elliott, M. G. Tehrani, and R. S. Langley, Nonlinear damping and quasi-linear modelling, Philos. Trans. R. Soc. A 373, 20140402 (2015).

[55] J. Atalaya, T. W. Kenny, M. L. Roukes, and M. I. Dykman, Nonlinear damping and dephasing in nanomechanical systems, Phys. Rev. B 94, 195440 (2016).

[56] M. Amabili, Derivation of nonlinear damping from viscoelasticity in case of nonlinear vibrations, Nonlinear Dyn. 97, 1785 (2019).

[57] N. Inomata, K. Saito, and T. Ono, Q factor enhancement of Si resonator by nonlinear damping, Microsyst. Technol. 23, 1201 (2017).

[58] M. I. Dykman, G. Rastelli, M. L. Roukes, and E. M. Weig, Resonantly Induced Friction and Frequency Combs in Driven Nanomechanical Systems, Phys. Rev. Lett. 122, 254301 (2019).

[59] N. E. Bousse, J. M. L. Miller, A. L. Alter, C. P. Cameron, H.-K. Kwon, G. D. Vukasin, and T. W. Kenny, Negative nonlinear dissipation in microelectromechanical beams, J. Microelectromech. Syst. 29, 954 (2020).

[60] X. Dong, M. I. Dykman, and H. B. Chan, Strong negative nonlinear friction from induced two-phonon processes in vibrational systems, Nat. Commun. 9, 3241 (2018).

[61] S. I. Lee, S. W. Howell, A. Raman, and R. Reifenberger, Nonlinear dynamics of microcantilevers in tapping mode atomic force microscopy: A comparison between theory and experiment, Phys. Rev. B 66, 115409 (2002).

[62] H. Hölscher, D. Ebeling, and U. D. Schwarz, Theory of Qcontrolled dynamic force microscopy in air, J. Appl. Phys. 99, 084311 (2006).

[63] P. G. Steeneken, K. Le Phan, M. J. Goossens, G. E. J. Koops, G. J. A. M. Brom, C. van der Avoort, and J. T. M. van Beek, 
Piezoresistive heat engine and refrigerator, Nat. Phys. 7, 354 (2011).

[64] J. M. L. Miller, H. Zhu, D. B. Heinz, Y. Chen, I. B. Flader, D. D. Shin, J. E.-Y. Lee, and T. W. Kenny, Thermal-Piezoresistive Tuning of the Effective Quality Factor of a Micromechanical Resonator, Phys. Rev. Applied 10, 044055 (2018).

[65] C.-C. Chu, S. Dey, T.-Y. Liu, C.-C. Chen, and S.-S. Li, Thermal-piezoresistive SOI-MEMS oscillators based on a fully differential mechanically coupled resonator array for mass sensing applications, J. Microelectromech. Syst. 27, 59 (2018).

[66] E. Mehdizadeh, V. Kumar, and S. Pourkamali, Sensitivity enhancement of Lorentz force MEMS resonant magnetometers via internal thermal-piezoresistive amplification, IEEE Electron Dev. Lett. 35, 268 (2013).

[67] A. Ramezany and S. Pourkamali, Ultrahigh frequency nanomechanical piezoresistive amplifiers for direct channel-selective receiver front-ends, Nano Lett. 18, 2551 (2018).

[68] R. N. Candler, W.-T. Park, H. Li, G. Yama, A. Partridge, M. Lutz, and T. W. Kenny, Single wafer encapsulation of MEMS devices, IEEE Trans. Adv. Packag. 26, 227 (2003).

[69] Y. Yang, E. J. Ng, Y. Chen, I. B. Flader, and T. W. Kenny, A unified epi-seal process for fabrication of high-stability microelectromechanical devices, J. Microelectromech. Syst. 25, 489 (2016).

[70] J. H. Comtois and V. M. Bright, Applications for surfacemicromachined polysilicon thermal actuators and arrays, Sens. Actuators A 58, 19 (1997).

[71] Q.-A. Huang and N. K. S. Lee, Analysis and design of polysilicon thermal flexure actuator, J. Micromech. Microeng. 9, 64 (1999).

[72] B. V. Derjaguin, V. M. Muller, and Y. P. Toporov, Effect of contact deformations on the adhesion of particles, J. Colloid Interface Sci. 53, 314 (1975).

[73] U. D. Schwarz, A generalized analytical model for the elastic deformation of an adhesive contact between a sphere and a flat surface, J. Colloid Interface Sci. 261, 99 (2003).

[74] See Supplemental Material at http://link.aps.org/supplemental/ 10.1103/PhysRevResearch.3.033268 for a discussion of the displacement calibration and additional measurements and simulations of the nonlinear-damping-stabilized oscillators.
[75] C. L. Muhlstein, S. B. Brown, and R. O. Ritchie, High-cycle fatigue of single-crystal silicon thin films, J. Microelectromech. Syst. 10, 593 (2001).

[76] V. A. Hong, S. Yoneoka, M. W. Messana, A. B. Graham, J. C. Salvia, T. T. Branchflower, E. J. Ng, and T. W. Kenny, Fatigue experiments on single crystal silicon in an oxygen-free environment, J. Microelectromech. Syst. 24, 351 (2014).

[77] E.-C. Chang, C.-C. Chen, and S.-S. Li, Real-time mass sensing and dynamic impact monitoring of printed picoliter droplets realized by a thermal-piezoresistive selfsustained oscillator, in 2016 IEEE 29th International Conference on Micro Electro Mechanical Systems (MEMS), pp. 1078-1081, (IEEE, New York, 2016).

[78] H. K. Kwon, Ultra Low Power Oven Controlled MEMS Resonator Timing Reference, Ph.D. thesis, Stanford University, 2020.

[79] M. Sansa, E. Sage, E. C. Bullard, M. Gély, T. Alava, E. Colinet, A. K. Naik, L. G. Villanueva, L. Duraffourg, M. L. Roukes et al., Frequency fluctuations in silicon nanoresonators, Nat. Nanotechnol. 11, 552 (2016).

[80] G. Lehée, R. Anciant, F. Souchon, A. Berthelot, P. Rey, and G. Jourdan, P-Type Silicon Nanogauge Based Self-Sustained Oscillator, in 2017 19th International Conference on Solid-State Sensors Actuators Microsystems (IEEE, Piscataway, NJ, 2017), pp. 444-447.

[81] L. C. Ortiz, H.-K. Kwon, J. Rodriguez, Y. Chen, G. D. Vukasin, D. B. Heinz, D. D. Shin, and T. W. Kenny, Low-power dual mode MEMS resonators with ppb stability over temperature, J. Microelectromech. Syst. 29, 190 (2020).

[82] J. M. L. Miller, N. E. Bousse, D. B. Heinz, H. J. K. Kim, H.-K Kwon, G. D. Vukasin, and T. W. Kenny, Thermomechanicalnoise-limited capacitive transduction of encapsulated MEM resonators, J. Microelectromech. Syst. 28, 965 (2019).

[83] H. K. Lee, R. Melamud, S. Chandorkar, J. Salvia, S. Yoneoka, and T. W. Kenny, Stable operation of MEMS oscillators far above the critical vibration amplitude in the nonlinear regime, J. Microelectromech. Syst. 20, 1228 (2011).

[84] S. C. Chapra, Applied Numerical Methods with MATLAB for Engineers and Scientists, (McGraw-Hill, New York, 2012). 